\title{
Current development of biodegradable polymeric materials for biomedical applications
}

This article was published in the following Dove Press journal:

Drug Design, Development and Therapy

\author{
Richard Song' \\ Maxwell Murphy' \\ Chenshuang $\mathrm{Li}^{\prime}$ \\ Kang Ting ${ }^{1-3}$ \\ Chia Soo ${ }^{2}$ \\ Zhong Zheng' \\ 'Division of Growth and \\ Development, Section of \\ Orthodontics, School of Dentistry, \\ University of California, Los \\ Angeles, Los Angeles, CA, USA; \\ ${ }^{2} U C L A$ Department of Surgery and \\ Department of Orthopaedic Surgery \\ and The Orthopaedic Hospital \\ Research Center, University of \\ California, Los Angeles, Los Angeles, \\ CA, USA; ${ }^{U}$ UCLA Department of \\ Bioengineering, School of Engineering, \\ University of California, Los Angeles, \\ Los Angeles, CA, USA
}

Correspondence: Chia Soo

UCLA Department of Surgery and

Department of Orthopaedic Surgery

and The Orthopaedic Hospital Research

Center, University of California, 675

Charles E Young Drive, South MRL 264I,

Los Angeles, CA 90095-1759, USA

Tel + I 3 I0 7945479

Fax + I 310 2067783

Email bsoo@ucla.edu

\section{Zhong Zheng}

Division of Growth and Development,

Section of Orthodontics, School of

Dentistry, University of California, 675

Charles E Young Drive, South MRL 264I,

Los Angeles, CA 90095-1759, USA

Tel +I 3102065646

Fax + I 3102067783

Email zzheng@dentistry.ucla.edu

\begin{abstract}
In the last half-century, the development of biodegradable polymeric materials for biomedical applications has advanced significantly. Biodegradable polymeric materials are favored in the development of therapeutic devices, including temporary implants and three-dimensional scaffolds for tissue engineering. Further advancements have occurred in the utilization of biodegradable polymeric materials for pharmacological applications such as delivery vehicles for controlled/sustained drug release. These applications require particular physicochemical, biological, and degradation properties of the materials to deliver effective therapy. As a result, a wide range of natural or synthetic polymers able to undergo hydrolytic or enzymatic degradation is being studied for biomedical applications. This review outlines the current development of biodegradable natural and synthetic polymeric materials for various biomedical applications, including tissue engineering, temporary implants, wound healing, and drug delivery.
\end{abstract}

Keywords: tissue engineering, drug delivery, wound healing, natural biomaterials, synthetic biomaterials

\section{Introduction}

A biomaterial can be defined as a material intended to interface with biological systems in order to evaluate, treat, augment, or replace any tissue, organ, or function of the body. ${ }^{1}$ The global market for implantable biomaterials was worth nearly $\$ 75.1$ billion in 2013 . This market is expected to grow at a compound annual growth rate (CAGR) of $6.7 \%$ between 2014 and 2019, resulting in a $\$ 79.1$ billion global market in 2014 and a $\$ 109.5$ billion global market in $2019 .^{2}$ Although biomedical applications of natural enzymatically degradable polymers date back thousands of years, the application of synthetic biodegradable polymers began only in the second half of the 1960s. ${ }^{3}$ Considering their advantages over biostable materials in terms of long-term biocompatibility along with the technical and ethical issues accompanying revision surgeries, investigations into the application of biodegradable biomaterials rather than permanent prosthetic devices for assisting in tissue repair and regeneration has vigorously increased recently. ${ }^{4-6}$ As a result, polymeric biomaterials are quickly replacing other material classes, such as metals, alloys, and ceramics, for use as biomaterials due to their versatility. ${ }^{3-5}$ Within the global implantable biomaterial market, the polymeric biomaterials sector is expected to show the highest growth, at a CAGR of $22.1 \%$, because of its promising potential in a wide range of biomedical applications. ${ }^{2}$ With this in mind, the aim of this review is to highlight the most often studied polymeric biomaterials and underscore their immense potential in the areas of drug design, development, and therapy. submit your manuscript

Dovepress

f

http://dx.doi.org/10.21471DDOTS165440
Drug Design, Development and Therapy 20|8:12 3117-3/45

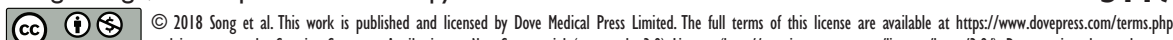
cc. hereby accept the Terms. Non-commercial uses of the work are permitted without any further permission from Dove Medical Press Limited, provided the work is properly attributed. For permission for commercial use of this work, please see paragraphs 4.2 and 5 of our Terms (https://www.dovepress.com/terms.php). 
A critical requirement for a biomaterial is biocompatibilitythe ability of a material to function with an appropriate host response in a specific application. ${ }^{1}$ Many biological and physicochemical characteristics of an implant material govern the host tissue response to the material. For instance, molecular weight, solubility, hydrophilicity/hydrophobicity, surface energy, material chemistry, mechanism of degradation and/or erosion, lubricity, and shape and structure of the implant can all influence the material's biocompatibility. ${ }^{\text {? }}$ Importantly, a biodegradable biomaterial requires excellent biocompatibility over time because the physicochemical, mechanical, and biological properties of a biodegradable biomaterial will differ with time and, thus, the resulting degradation products can possess varying levels of tissue compatibility as compared with the initial parent material. An ideal biodegradable biomaterial should have degradation products that are nontoxic and easily metabolized and cleared from the body.

In addition to biocompatibility, several other important properties must be considered when choosing a biodegradable biomaterial. First, the degradation time of the biomaterial should coincide with the regeneration and/or healing process to ensure proper remodeling of the tissue. Second, the biomaterial must maintain suitable permeability and processability for its intended application. Finally, the mechanical properties of the biomaterial should be sufficient to promote regeneration during the patient's everyday activities, and any change in mechanical properties due to degradation should preserve compatibility with the healing or regeneration process.

Given the complexity of the human body and the scope of applications that polymeric biomaterials are currently utilized for, no single polymeric system can be considered the ideal biomaterial for all medical applications. Thus, recent advances in biodegradable biomaterial synthesis have been directed toward developing and synthesizing polymers with properties tailored for specific biomedical applications. Moreover, current developments incorporating multifunctional and combinatorial approaches in biomaterial design have accelerated the innovation of novel biodegradable biomaterials. Another hotspot in biomaterials research is the development of therapeutic devices, including temporary prostheses, three-dimensional (3D) porous scaffolds for tissue engineering, and delivery vehicles for pharmacological applications. Most recently, 3D bioprinting has also been acknowledged, and preliminary data collection for biomaterial use as potential bio-ink for printing of 3D scaffolds have begun. Because biodegradable biomaterials exhibit a variety of biological and physicochemical properties and, therefore, can replicate the properties of different tissues, these materials are assessed for use as 1) large implants, including bone screws, bone plates, and contraceptive reservoirs; 2) small implants, in the form of sutures and staples; 3 ) plain membranes for guided tissue regeneration; and 4) porous structures or multifilament meshes for tissue engineering. ${ }^{8}$ Moreover, by properly engineering the structure and degradation parameters, these biodegradable materials can be used to generate micro- or nanoscale drug-delivery vehicles for controlled drug delivery in an erosive or diffusive manner, or as a combination of both. ${ }^{9}$

Because of the interest aroused in the areas demanding biodegradable biomaterials, including regenerative medicine, tissue engineering, controlled drug delivery, gene therapy, and nanotechnology, there has been a robust expansion of biomedical applications of synthetic biodegradable polymers and analogous natural polymers, and our review will focus on exploring the most current development of these polymers for biomedical applications in these fields. Although many other reviews have focused on the topic of biodegradable biomaterials for medical use, to our best knowledge, there has not been a review published within the past 5 years that covers as much breadth as our review does about the development of the most commonly investigated biodegradable polymeric biomaterials in the fields of drug delivery, tissue engineering, and wound application.

\section{Natural biodegradable polymeric biomaterials}

Biodegradable biomaterials can be roughly divided into two categories - natural and synthetic - based on their source and whether they are composed of naturally occurring extracellular matrix (ECM). Natural biodegradable polymeric biomaterials generally include proteins (collagen, fibrin, silk, etc.), and polysaccharides (starch, alginate, chitin/chitosan, hyaluronic acid derivatives, etc.). ${ }^{10-12}$ Furthermore, a family of native polyesters - polyhydroxyalkanoates (PHA) - has been recognized as natural biodegradable biomaterials and, more recently, sundew adhesives (natural polysaccharidebased hydrogels) and ivy nanoparticles (macromolecular compositions of nanospherical arabinogalactan proteins) have garnered more attention for their ability to create effective nanocomposite adhesives and for their potential use as nano-carriers in drug delivery, respectively. ${ }^{13,14}$

\section{Collagen}

As the most prevalent protein in the human body, collagen offers physical support to tissues by inhabiting the intercellular space, acting not only as native structural support for 
organizing cells within connective tissues, but also as a mobile, dynamic, and flexible substance essential to cellular behaviors and tissue function. ${ }^{15}$ Generally, collagen is a rodtype polymer approximately $300-\mathrm{nm}$ long with a molecular weight of approximately $300 \mathrm{kDa}$. Free amino acids in the body are synthesized into subunit chains of collagen, which then undergo transcription, translation, and post-translational modification processes in suitable cells such as osteoblasts and fibroblasts. ${ }^{13}$ More than 22 different types of collagen have been identified in the human body, with types I-IV being the most common, and type I collagen being the single greatest abundant protein present in mammals. ${ }^{15}$ Collagen undergoes enzymatic degradation within the body by a diverse set of enzymes, such as matrix metalloproteinases and collagenases, to yield its corresponding amino acids. Due to its enzymatic degradability; unique mechanical, biological, and physicochemical properties; nontoxicity; and high tensile strength, collagen has been widely studied for biomedical applications. ${ }^{16,17}$

Collagen plays a critical role in preserving the biological and structural integrity of the ECM and is highly dynamic, undergoing continuous remodeling for proper physiologic functions. For most soft and hard connective tissues (eg, blood vessels, cornea, skin, tendon, cartilage, and bone), collagen fibrils and their networks function through their highly organized 3D structure. Tissue regeneration attempts to repair both the structural integrity and the intricate remodeling process of the native ECM, particularly restoring the delicate collagen networks under which normal physiologic regeneration occurs; thus, recent efforts have been focused on replacing native collagen-based ECM by developing novel biomaterials that imitate its intricate fibrillar architecture and function as cell scaffolding. Animal-derived and recombinant collagens, especially type I collagen, are recognized as one of the most valuable biomaterials available and are now extensively used for tissue engineering, drug delivery, and cosmetic surgery. For example, a composite of fibrillar collagen, hydroxyapatite, and tricalcium phosphate (Collagraft $^{\circledR}$, Angiotech Pharmaceuticals) has been approved by the United States Food and Drug Administration (FDA) as a biodegradable synthetic bone graft substitute. ${ }^{18}$

The main sources of collagen presently utilized for biomedical applications are bovine or porcine skin and bovine or equine Achilles tendons. They are utilized in either their native fibrillar form or after denaturation in various fabricated forms, such as sponges, sheets, plugs, and pellets. Collagenbased materials have been successfully used for skin repair. ${ }^{19}$ For example, Promogran ${ }^{\circledR}$ (Systagenix) - a spongy collagen matrix containing oxidized cellulose - is available in the USA and Europe for treatment of diabetic and ulcer wounds. ${ }^{20}$ Similarly, an FDA-approved bilayer skin substitute (Integra ${ }^{\circledR}$ Dermal Regeneration Template, Integra LifeSciences) composed of a dermal layer of cross-linked bovine collagen and glycosaminoglycans (GAGs) as well as an epidermal layer of polysiloxane, which deposits ECM components, is in the market for full-thickness or deep partial thickness thermal injury. Moreover, these skin substitutes constructed from cell-seeded collagens have been widely commercialized (eg, Apligraf ${ }^{\circledR}$, Organogenesis, Inc.) and $\left(\mathrm{OrCel}^{\mathrm{TM}}\right.$, Ortec International Inc.). Interestingly, type I collagen sponges have also been used to engineer patellar tendons in rabbits under different culture conditions. ${ }^{21-23}$ Using the collagen sponge combined with bone marrow-derived mesenchymal stem cells, Juncosa-Melvin et al demonstrated that the engineered tendon tissue attained almost $75 \%$ of the mechanical properties of native tendon. ${ }^{22}$ In other studies, collagen has also been successfully used as a scaffold in nerve and bladder engineering. ${ }^{24-26}$ Additionally, a suture-free, 3D-collagen matrix graft - DuraGen ${ }^{\circledR}$ (Integra LifeSciences) - has been developed for spinal dural repair and regeneration and is currently approved by the FDA. ${ }^{27}$

Collagen is, furthermore, a key initiator of the coagulation cascade and, therefore, it has been successfully developed as a hemostatic agent due to its high thrombogenicity. Multiple collagen-based hemostats are currently available or undergoing clinical trials for a variety of surgical indications; these include Sulzer-Spine ${ }^{\circledR}$ Tech's sealants composed of bovine collagen and bovine thrombin for cardiovascular and spinal surgical procedures; Floseal ${ }^{\circledR}$ (Baxter Healthcare), a highviscosity gel hemostatic agent composed of collagen-derived particles and topical bovine-derived thrombin; and CoStasis ${ }^{\circledR}$ Surgical Hemostat (Cohesion Technologies), which consists of bovine thrombin and bovine microfibrillar collagen combined with autologous plasma.

With regard to drug delivery, collagen has been notably studied for the delivery of low-molecular-weight drugs, proteins, genes, and plasmids. Currently, a few collagenbased gentamicin-delivery vehicles are available in the global market (eg, Sulmycin ${ }^{\circledR}$-Implant and Collatamp ${ }^{\circledR}-\mathrm{G}$, Innocoll Pharmaceuticals Ltd). These delivery systems permit a sustained local delivery of antibiotics with limited systemic exposure. Moreover, another product, Septocoll ${ }^{\circledR}$ (Biomet), achieved prolonged collagen delivery by incorporating two gentamicin salts possessing different solubility, and has been approved for infection prevention. ${ }^{28}$ More recently, a new biodegradable, collagen-based chlorhexidine chip has been shown to provide a longer, more sustained release of chlorhexidine in the confines of the periodontal pocket as 
compared to simple subgingival irrigation of chlorhexidine. The constant outflow of the gingival crevicular fluid from the periodontal pocket (up to 40 times an hour) renders subgingival irrigation of chlorhexidine useless in delivering significant antimicrobial benefits, reduction in probing depths, and clinical attachment level gain to manage chronic periodontitis; however, the use of a collagen-based release vehicle allows for a controlled delivery of chlorhexidine to achieve the intended pharmaceutical effects. ${ }^{29}$ Furthermore, current clinical trials are investigating cross-linked, absorbable collagen sponges as protein-carrier vehicles. Prolonged release of bioactive proteins, such as recombinant human bone morphogenic protein-2 (rhBMP-2), have been reported when utilized in conjunction with collagen matrices due to desirable interactions of the collagen matrix with the protein. ${ }^{30}$ This combination product is approved by the FDA for simultaneous use with a titanium interbody spine fusion cage for anterior lumbar spinal fusion (InFUSE ${ }^{\circledR}$ Bone Graft/LT-CAGE ${ }^{\circledR}$ Lumbar Tapered Fusion Device, Medtronic Spinal and Biologics), and a similar product InductOs $^{\circledR}$ (Medtronic Spinal and Biologics) is approved in Europe for the treatment of acute tibial fractures in adult patients. Additionally, a recent study reported that the delivery of rhBMP-2 by an absorbable collagen sponge stimulated bone reconstruction in advanced alveolar ridge defects. ${ }^{31}$ Aside from its use as a protein-delivery vehicle, collagen has been demonstrated to retain gene vector/plasmid DNA whereas simultaneously protecting it from enzymatic or immunological reactions of the body, which underscores its potential in gene and plasmid DNA delivery. ${ }^{32}$

One disadvantage of these collagen-based biomaterials is their mild immunogenicity due to the antigenic sites in the central helix and the composition of the terminal region, which significantly limits their clinical application. ${ }^{33}$ The immune response varies depending on the processing techniques, site of implantation, and species from which the collagen has been isolated. ${ }^{34}$ Other disadvantages of animal-derived collagen include the varying physicochemical and degradation properties, the high cost of pure collagen, and the allogeneic or xenogeneic sources, which increase the risk of infectious disease transmission. In response to these limitations, present investigations have directed focus toward recombinant systems that can produce human-based collagen. ${ }^{35,36}$ Several potentially useful systems already exist for large-scale production and purification of recombinant collagen, such as yeasts, transgenic animals, and, most recently, Escherichia coli. ${ }^{37,38}$ Because the amino acid sequence of recombinant collagen can be directly modified, it is possible to make controlled, targeted collagen products for specific applications, thereby diversifying and increasing the potential of collagen-based products. ${ }^{39}$ However, recombinant collagen is unable to undergo natural posttranslational modification and, thus, may lack critical biological activities of native tissue. ${ }^{33}$ This lack of posttranslational modification means that, despite its flexibility in creating countless different amino acid sequences for different targeted applications, recombinant collagens may not be suitable for many biomedical applications, especially those that require stability and strength such as heart valves. Furthermore, the commercially available recombinant collagens are still expensive to produce and, thus far, only limited amounts are available. ${ }^{39}$

Collagen as a biomaterial has already seen significant use in several specific applications in skin repair, hemostatic agents, and drug delivery, but its slight immunogenicity, high cost, and varying physicochemical and degradation properties prevent further expansion of collagen-based biomaterials. Although recombinant collagens have the potential to catapult collagen-based biomaterials for widespread use, significant limitations such as their lack of posttranslational modification will need to be continually studied for them to have any noticeable impact for use as a biomaterial.

\section{Gelatin}

Gelatin is a natural biopolymer derived from collagen via controlled alkaline, acid, or enzymatic hydrolysis. ${ }^{40}$ As a result of its biological origin, it has excellent biodegradability and biocompatibility and because it is widely available, gelatin is a relatively low-cost polymer. ${ }^{40}$ Gelatin has been used in medical and pharmaceutical fields as a matrix for implants, and as stabilizers in vaccines such as measles, mumps, and rubella. ${ }^{41}$ Moreover, gelatin is water permeable and soluble in water and has multifunctional properties as a drug-delivery carrier. ${ }^{42}$ Gelatin's mechanical properties, swelling behavior, thermal properties, and many other physiochemical properties can be dependent upon the collagen source, extraction method, amount of thermal denatured employed, and the degree of cross-linking, thereby making gelatin a very versatile polymer. ${ }^{43}$ Furthermore, its ability to produce a thermoreversible gel makes it a very good candidate as a targeted drug-delivery carrier and, as a result, gelatin can be utilized to develop specific drug-release profiles, allowing for a broad range of applications in drug delivery. ${ }^{43}$

Gelatin is a versatile biopolymer that traditionally enabled the design of several different drug carrier systems, such as microparticles, nanoparticles, fibers, and hydrogels. ${ }^{44}$ Each of these different systems has certain properties that make 
them particularly suitable for drug delivery. ${ }^{44}$ For example, gelatin microparticles are popular for serving as vehicles for cell amplification and delivery of large bioactive molecules, and gelatin nanoparticles are better for drug delivery to the brain or in intravenous delivery. ${ }^{44}$ Recently, a novel and relatively simple method was discovered for producing gelatin microparticles which allow for very high protein- and drug-loading efficiencies. ${ }^{45} \mathrm{In}$ the experiment, bovine serum albumin (BSA) was added at a $0.1 \%-0.4 \%$ gelatin concentration, resulting in a gelatin solution. ${ }^{45}$ The solution was freezedried and the resulting spongy membranes were hardened with liquid nitrogen and subsequently ground to a powder, producing BSA-loaded microparticles in random forms and shapes. ${ }^{45}$ These microparticles were then incorporated into porous scaffolds constructed of polylactide (PLLA) and polycaprolactone (PCL) ${ }^{45}$ The in vitro release profile of the protein from the particles alone and from the particle-incorporated scaffolds demonstrated up to $90 \%$ protein-loading efficiency, showing that BSA-loaded microparticles in conjunction with particle-incorporated scaffolds for growth factor release may be possible for application in bone tissue engineering. ${ }^{45}$ Another study studied the in vitro efficacy and toxicity of incorporating polyenes into electrospun gelatin fiber mats as a topical antifungal application. ${ }^{46}$ The research group found that polyene-loaded antifungal gelatin mats displayed better antifungal activity than using traditional electrospun gelatin fiber mats. ${ }^{46}$ Furthermore, they found that polyenes stabilized the triple helical conformation of gelatin whereas gelatin decreased the hemolytic activity of polyenes, making polyene antifungal-loaded gelatin fiber mats a possibility in managing superficial skin infections in the future ${ }^{46}$ Cohen et al studied novel tissue adhesives based on gelatin, with alginate as a polymeric additive, loaded with bupivacaine or ibuprofen for pain management. ${ }^{47}$ They found that the release of drugs from the adhesive matrix was primarily controlled by the gelatin/alginate bioadhesive's characteristics, such as swelling and hydrophilic group concentrations that can be adjusted by changing the ratio of gelatin to alginate. ${ }^{47}$ Moreover, they found that the hydrophilicity and electrical interactions between bupivacaine and ibuprofen with the adhesive components had some effect on the release profile of the drug, and that incorporating bupivacaine improved the bonding strength of the adhesive due to its inert nature whereas ibuprofen decreased the bonding strength due to its reactive nature with the alginate/gelatin adhesive. ${ }^{47}$ Overall, the study shows that this loading bupivacaine in a gelatin/ alginate bioadhesive can potentially be used for woundclosing applications. ${ }^{47}$
The primary advantages of gelatin are its biodegradability, availability, and cheap cost. ${ }^{44}$ Porcine skin-derived gelatin is the most popular source, followed by bovine skin, and bone ${ }^{48}$ However, religious issues may arise, as porcinederived products and bovine-derived products are forbidden in Judaism and Hinduism, respectively ${ }^{48}$ In addition, there are health concerns over transmission of pathogenic vectors such as prions. ${ }^{48}$ Recombinant gelatins, however, can be utilized to overcome the disadvantages with animal tissuederived materials ${ }^{49}$ Currently, the primary use of gelatin is in the food, pharmaceutical, and photographic industries. ${ }^{49}$ In the biomedical field, gelatin is used in drug delivery and in some wound dressing and tissue-regeneration applications, but its use is heavily limited by its poor mechanical properties. ${ }^{49}$ These mechanical properties can be strengthened through physical cross-linking as well as chemical crosslinking due to a large number of functional side groups that gelatin possesses; however, agents used to stabilize crosslinked gelatin are often somewhat toxic to the human body ${ }^{49}$ Research into improving gelatin's mechanical properties will need to be investigated in the future for gelatin to have a promising future as a primary biomaterial; but, until then, the most likely incorporation of gelatin will be as a composite with other natural or synthetic biomaterials, or as a carrier for drug delivery.

\section{Fibrin}

Fibrin is a $360-\mathrm{kD}$ fibrinogen-derivative biopolymer involved in the natural blood-clotting process, enhancing cell adhesion and proliferation. ${ }^{50}$ In addition to possessing excellent biodegradability and biocompatibility, fibrin exhibits high elastic and viscous properties; stiffens in response to shear, tension, or compression; and has excellent deformability. ${ }^{51}$ One of the first developed fibrin-based products was fibrin glue (fibrin sealant). Tissucol/Tisseel ${ }^{\mathrm{TM}}$ (Baxter Healthcare) and Beriplast HS/Beriplast PTM (CSL Behring) are the firstgeneration products of fibrin sealants marketed in Europe. Today, an expansive variety of products possessing different compositions and adhesive properties are available on the market. These products are broadly used for hemostasis and tissue-sealing applications in various surgical procedures, including neurosurgery and plastic and reconstructive surgery. ${ }^{52-54}$

Furthermore, fibrin has been utilized as a scaffold for the regeneration of numerous tissues, such as adipose tissue, bone, cardiac tissue, cartilage, muscle tissue, nervous tissue, ocular tissue, respiratory tissue, skin, tendons and ligaments, and vascular tissue as well as a carrier vehicle 
for bioactive molecules (drugs, antibiotics, or chemotherapy agents) due to its injectability and biodegradability. ${ }^{55-60}$ For instance, several studies have demonstrated that clinical application of fibrin significantly improved the healing of intrabony defects in chronic periodontitis. ${ }^{61,62}$ Further evidence suggests that proteins interact differently with fibrin clots and, as a result, several cross-linking techniques are presently being studied to control the release profile of bioactive molecules from the fibrin matrix. ${ }^{63}$ Additionally, fibrin matrices have been utilized as excellent cell-carrier vehicles. One successful example obtained by mixing keratinocytes with fibrin is Bioseed ${ }^{\circledR}$ (DCM Shriram Limited), a fibrinbased product used to treat chronic cutaneous wounds.

Fibrin is natural, highly available, implantable, inexpensive, easy to use, and has low fibrinogen concentrations. Due to its porous morphology, a fibrin scaffold system is adequate for cell attachment, proliferation, differentiation, ${ }^{58,64}$ and as a release system of growth factors such as vascular endothelial growth factor and basic fibroblast growth factor (bFGF). ${ }^{60,65}$ Fibrin-immobilized growth factors have been shown to be continuously released for several days in a controlled manner, making it optimal for numerous tissue-engineering purposes. ${ }^{50}$ Thus, autologous fibrin could prevent the complications of techniques derived from the use of current commercial available fibrin products and should be further investigated. Fibrin-based scaffolds do have some limitations, such as weak mechanical strength and quick degradation rates; however, these properties have been shown to be improved by incorporating stronger natural and synthetic polymers, utilizing various cross-linking methods, and utilizing micro/nanospheres. ${ }^{50}$ Next-generation scaffolds will likely involve specific cell lines, combined with biomolecules and growth factors to accelerate and increase cell proliferation and differentiation on immobilized scaffolds for a variety of tissues, and fibrin-based drug-delivery carriers and tissue-engineered scaffolds, including the relatively recent scaffold development of fibrin microspheres, nanospheres, microfibers, microtubes, and porous sheets, all of which will play a big role in regenerative medicine..$^{50}$

\section{Hyaluronic acid (HA)}

HA is an essential component of the ECM and its structural and biological properties mediate cellular signaling, morphogenesis, matrix organization, and wound repair. ${ }^{66,67}$ In 1943, HA was first isolated from the vitreous humor by Meyer and Palmer. ${ }^{68} \mathrm{HA}$ is a member of the GAG family, which involves linear polysaccharides consisting of alternating units of $\mathrm{N}$-acetyl-d-glucosamine and glucuronic acid ranging in

\section{Hyaluronic acid}

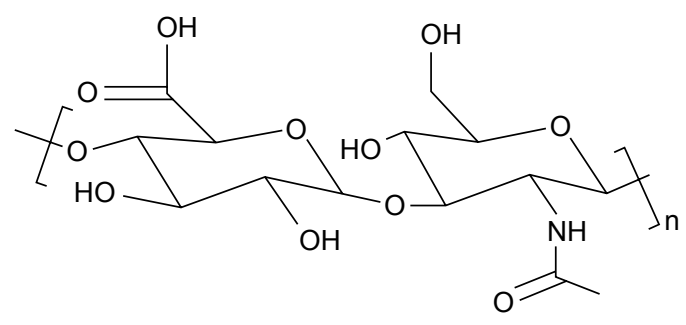

Figure I Structure of hyaluronic acid (HA).

size from 5,000 to 20,000,000 Daltons in vivo, and is present in almost every tissue in vertebrates. Native sources of HA include rooster combs, bovine vitreous humor, and synovial fluids. ${ }^{69} \mathrm{HA}$ degradation occurs in the body through free radicals, such as nitric oxide and matrix metalloproteinases found in the ECM, and then undergoes endocytosis. Further digestion of HA by lysosomal enzymes results in monoand disaccharides, which are then converted into ammonia, carbon dioxide, and water. ${ }^{69}$

Recently, HA has become recognized as an important building block for the creation of new biomaterials for use in cell therapy, 3D cell culture, and tissue engineering. ${ }^{70-72}$ As HA is secreted at the early stage of wound healing, it has been extensively researched for wound-dressing applications. ${ }^{73,74}$ HA can be recognized by receptors on a variety of cells associated with tissue repair, and thus presents the capability to stimulate angiogenesis and to regulate injury-induced inflammation as a free radical scavenger. ${ }^{75}$ Moreover, HA promotes epithelial and mesenchymal cell migration and differentiation, making it vital for tissue repair. ${ }^{75}$ These properties combined with its immunoneutral potency make $\mathrm{HA}$ an ideal biomaterial for tissue engineering. ${ }^{75}$ Moreover, its aqueous solubility allows for modification of HA into various porous and 3D structures for drug delivery. For example, HYAFF ${ }^{\circledR} 11$ (Anika Therapeutics, Inc.), an HAbased product, is currently utilized as a carrier vehicle for a variety of growth factors, morphogens, and stem cells. ${ }^{76}$ In a comparative study, Hunt et al reported an improved healing response to rhBMP-2 delivered by $\mathrm{HYAFF}^{\circledR} 11$ than that delivered by an absorbable collagen sponge. ${ }^{76}$ A more recent study indicated that HA-based materials maintain the potential to replace collagen-based materials as injectable soft tissue fillers. ${ }^{77}$ With regard to tissue engineering, HA has been successfully incorporated into multiple complex systems. For example, HA-modified poly $\left(_{\mathrm{D}, \mathrm{L}}\right.$-lactic acid-co-glycolic acid) (PLGA) scaffolds were successful in inducing cartilage tissue formation in terms of type II collagen expression and 
tissue morphological characteristics. ${ }^{78}$ Additionally, highmolecular-weight viscous HA solutions (eg, AMVISC ${ }^{\circledR}$ and AMVISC ${ }^{\circledR}$ PLUS, Bausch \& Lomb) currently function as vitreous humor substitutes and as protection for sensitive eye tissue during glaucoma surgery, cataract extraction, and corneal transplantation. Viscous HA solutions (eg, SYNVISC ${ }^{\circledR}$, SanofiBiosurgery; and ORTHOVISC ${ }^{\circledR}$, Anika Therapeutics, Inc.) are also clinically applied as synovial fluid substitutes to reduce pain and enhance joint mobility in patients with osteoarthritis. ${ }^{79}$

Moreover, HA derivatives, such as HA esters and crosslinked HA gels, have been thoroughly researched for wound dressing applications. Reports suggest that these chemical modifications significantly reduce the degradation of HA. ${ }^{69,80}$ For example, in the absence of enzymatic activity, benzyl HA esters undergo hydrolytic degradation via ester bonds, and the degradation time varies from 1 to 2 weeks to $2-3$ months depending on the degree of esterification. ${ }^{69,80}$ Together, these studies underscore the potential of HA in combined systems for an array of biomedical applications, including tissue engineering, drug delivery, wound healing, and temporary implants.

\section{Chitin/chitosan}

Chitosan is another naturally derived biodegradable polysaccharide commonly used in tissue engineering. ${ }^{81}$ Chitosan is a derivative of chitin - the second most abundant natural polymer commonly found in the exoskeletons of crustacean and insects as well as the cells walls of fungi. ${ }^{81}$ Chitin is partially deacetylated to form chitosan, which is composed of glucosamine and $N$-acetyl glucosamine linked in a $\beta(1-4)$ manner. ${ }^{82}$ The molecular weight and the degree of deacetylation, which are essential in assessing the characteristics of chitosan, are reliant on the source and production process.

Chitin and chitosan are biodegraded by human enzymes, such as lysozymes, which disrupt the linkage between acetylated units and degrades chitin/chitosan to oligosaccharides..$^{83}$ The degradability of chitin/chitosan-based materials is essential for scaffold construction because it can influence cell behavior and tissue formation of the engineered construct. ${ }^{84}$ However, the low mechanical resistance of chitosan makes it disadvantageous to be used as a supporting material in tissue engineering. ${ }^{85}$ To create a better mechanical profile, cross-linking agents are used with functional reactive groups to allow for bridges to be made between polymeric chains, optimizing the resistance and elasticity of chitosan membranes ${ }^{85}$ Further, chitin/chitosan have the ability to chelate with the $\mathrm{Ca}^{2+}$ or $\mathrm{Mg}^{2+}$ present in the cell wall of bacteria, thus

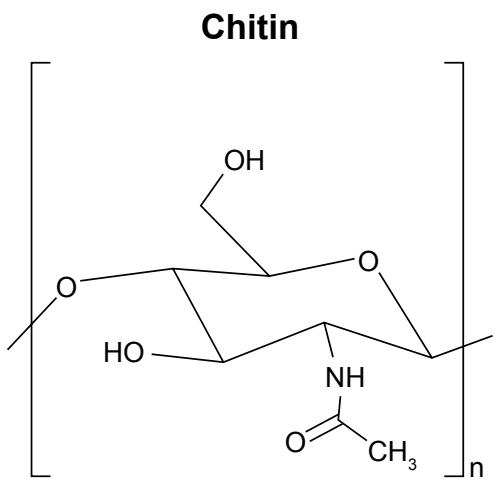

Chitosan

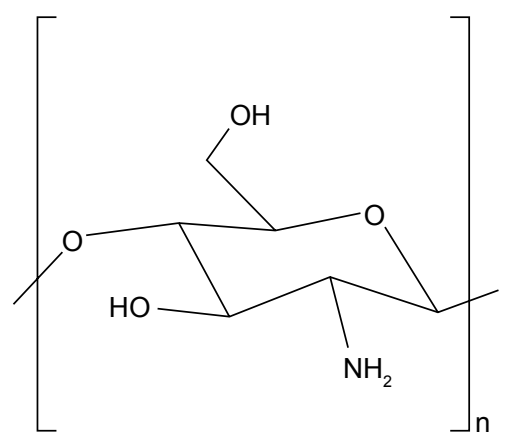

Figure 2 Structure of chitin and chitosan.

destroying the entity of bacterial cell wall, and to react with the anionic phosphate groups of phospholipids found on the bacterial cell membrane by using their $\mathrm{NH}_{3}^{+}$amino group, thereby leading to changes in the cell membrane permeability and eventual release of the bacteria's cellular contents. ${ }^{84,86}$ These functional capabilities make chitin/chitosan-based materials exhibit a bactericidal effect on both Gram-negative and Gram-positive bacteria, giving them a broad medical utility in tissue engineering and biomedical applications..$^{84,86}$

Specifically, chitin/chitosan-based materials have demonstrated potential with regard to connective, nerve, adipose, and vascular tissue-engineering applications. ${ }^{87-89}$ For example, a silk fibroin (SF)/chitin-based scaffold was used to repair a musculofascial defect in the abdominal wall, displaying continuous integration with adjacent native tissue and mechanical strength similar to native tissue.$^{88}$ Similarly, chitosan/hydroxyapatite-based scaffolds loaded with bFGF for periodontal tissue regeneration promoted vigorous proliferation and migration in periodontal ligamental cells and cementoblasts. ${ }^{87} \mathrm{~A}$ more recent study produced nanosized chitosan/hydroxyapatite-based scaffolds via thermally induced phase separation and lyophilization techniques and found that this combination demonstrated greater compressed mechanical properties as compared to the pure chitosan scaffold - a property critical for the success of scaffolds 
to be used as tissue-engineering scaffolds. ${ }^{90}$ Further tests will need to be conducted, but it is expected that this scaffold will allow cell attachment and tissue growth in vivo as well. Taken together, these results highlight the potential of chitin/chitosan-based scaffolds providing a cell-favorable microenvironment for connective tissue healing.

Though chitosan has been widely used for bone tissueengineering applications due to its various favorable properties as mentioned earlier, it has a lower tensile strength and modulus range than those of natural bones. ${ }^{91,92}$ In an effort to reinforce the mechanical properties of chitosan, Tamburaci et al combined diatomite (diatomaceous earth), a natural silica material, with chitosan to produce a scaffold for bone tissue regeneration; they found that diatomite-reinforced chitosan composite membranes improved the surface area, roughness, swelling properties, and protein adsorption capacities of traditional chitosan membranes, while showing no cytotoxic effect in Saos-2 osteosarcoma cell line with excellent biocompatibility. ${ }^{93}$ Interestingly, Tamburaci et al also found that diatomite incorporation into chitosan scaffolds increased the proliferation and alkaline phosphatase activity of Saos-2 cells remarkably. ${ }^{93}$ Overall, diatomitereinforced chitosan scaffolds showed improved properties while maintaining the high biological activity typical of traditional chitosan scaffolds, illustrating the potential of chitosan for bone tissue-engineering applications.

Moreover, glutaraldehyde-cross-linked collagen-chitosan hydrogels were successfully applied to adipose tissue engineering. A study confirmed the in vitro viability of preadipocytes (PAs) on glutaraldehyde-cross-linked collagenchitosan hydrogel scaffolds. Subsequently, a rat subcutaneous pocket assay was used to evaluate PA-seeded scaffolds in vivo which displayed excellent biocompatibility, formed adipose tissue, and induced vascularization. ${ }^{89}$ Additionally, chitin/chitosan-based materials have found success as biomaterials used in cartilage repair due to their biocompatibility and structural similarity with GAGs found in cartilage. ${ }^{12,94,95}$ Recently, Lee's group synthesized an injectable hydrogel consisting of methacrylated glycol chitosan and HA by photo-cross-linking with a riboflavin photo-initiator under visible light. ${ }^{12}$ This resulted in the formation of a cross-linked chitosan network in which high-molecular-weight HA was entrapped, forming a semi-interpenetrating network that provided a more chondrogenic-favorable microenvironment. ${ }^{12}$ Likewise, these photopolymerizable hydrogels exhibit robust capacity as cell carrier vehicles and further emphasize the advantage of chitin/chitosan-based materials for tissue engineering.
Furthermore, chitin/chitosan-based materials have been incorporated successfully into cutaneous wound management. A number of studies have reported the use of chitin/ chitosan scaffolds and membranes to treat patients with deep burns. ${ }^{96,97}$ Recently, novel $\alpha$-chitin/silver nanoparticles (AgNPs) and $\beta$-chitin/AgNP composite scaffolds were tested for wound-healing applications. ${ }^{96,97}$ These chitin/AgNPs composite scaffolds were found to possess excellent antibacterial activity against Staphylococcus aureus and E. coli, combined with good blood clotting ability. ${ }^{96,97}$ In addition, $\beta$-chitin/ AgNP composite scaffolds functioned as promising matrices capable of providing good cell attachment apart from their antibacterial activity, which suggests that these composite scaffolds are ideal for wound-healing applications. ${ }^{97}$

Finally, chitin/chitosan-based materials have presented excellent potential in drug delivery systems. For example, in one study, when carboxymethyl chitin nanoparticles were cross-linked with $\mathrm{FeCl}_{3}$ and $\mathrm{CaCl}_{2}$, they were shown to be nontoxic to mouse L929 cells while showing significant antibacterial activity against Staphylococcus strains. ${ }^{98}$ Studies indicating that chitin/chitosan-based materials may be used for controlled drug delivery in managing HIV are more exciting. In one study that focused on anti-transferrin and anti-bradykinin B2 antibody-conjugated chitosan nanoparticles, the investigators found that chitosan nanoparticles presented the potential to effectively penetrate across the blood-brain barrier and thus enhance the drug delivery in the brain to inhibit HIV replication in the neural system. ${ }^{99}$ Another study showed that using chitosan nanoparticles to encapsulate conventional antiretroviral drugs targeting HIV led to a more efficient control of the viral proliferation in target $\mathrm{T}$ cells. ${ }^{100}$ Greater cell targeting efficiency was achieved, mostly due to the fact that chitosan nanoparticles are mildly immunogenic, making them more visible to the immune system, allowing for more efficient uptake by phagocytes. ${ }^{100}$ A separate investigation studied the effects of poly(lactic acid) (PLA)/chitosan nanoparticles loaded with lamivudine (a type 1 and type 2 HIV selective inhibitor) in mouse L929 fibroblast cells. ${ }^{101}$ The in vitro drug-release studies showed that the drug release rate from PLA/chitosan nanoparticles decreased when the $\mathrm{pH}$ of the medium changed from alkaline to acidic and further decreased from acidic to neutral, which could be a result of the repulsion between $\mathrm{H}^{+}$ions and cationic groups present in the polymeric nanoparticles. ${ }^{101}$ Because it is ideal that the drug encapsulated in the delivery system will be protected in the stomach environment at acidic $\mathrm{pH}$ and then provide sustained release in the intestines (neutral $\mathrm{pH}$ ), these findings suggest that the 
chitosan-based nanoparticles exhibit excellent potential as a carrier system for HIV-controlled drug delivery.

Besides HIV management, chitin/chitosan-based materials can be used in cancer therapy as drug-delivery vehicles. For example, a pH-responsive magnetic nanocomposite was wrapped in chitosan for targeted and controlled drug delivery, and the yield product was found to be nontoxic and exhibited a high antitumor activity while maintaining its excellent $\mathrm{pH}$ sensitivity at $\mathrm{pH}<6.0 .^{102}$

In addition to improving the targeting and efficiency of cancer and HIV drug-delivery vehicles, chitin/chitosan-based materials have been utilized in various other delivery systems. In a very recent study, Di et al developed an ultrasoundtriggered insulin-delivery system which allows for pulsatile insulin release that can provide both long-term, sustained and fast on-demand responses. ${ }^{103}$ This system incorporated insulin-loaded PLGA nanocapsules encapsulated within chitosan microgels and, upon ultrasound treatment, the stored insulin can be rapidly released to regulate blood glucose levels. ${ }^{103}$ The research group found that in a mouse model of type 1 diabetes, a 30-second ultrasound administration could effectively achieve glycemic control for 1 week and concluded that this delivery system may potentially be used to release other therapeutics in a noninvasive and convenient manner. ${ }^{103}$ Moreover, Liang et $\mathrm{al}^{104}$ and Jing et al ${ }^{105}$ have shown that chitosan derivatives as a delivery system can also enhance oral tablet absorption of bioactive compounds and potentially allow protein and certain peptide drugs to be orally administrable. In addition, hydroxyethyl chitosan - a derivative of chitosan - has shown great potential as a drugdelivery material for the treatment of glaucoma and other ocular diseases due to its great water-solubility and excellent biocompatibility. ${ }^{106}$ Further investigations must be carried out to assess any side-effect or instability of chitosan-based materials; however, there seems to be substantial potential for chitosan-derived drug-controlled-release systems.

Chitin/chitosan-based materials are widely studied for several different tissue-engineering applications, regenerative medicine, wound healing, and drug delivery for good reason. They have excellent biodegradability and biocompatibility and has been known to have antiulcer, anti-acid, hypocholesterolemic action, wound-healing, antitumor, and hemostatic properties. ${ }^{107-110}$ Although chitin/chitosan-based materials tend to lack mechanical properties, they possess functional reactive side groups that can be cross-linked to make bridges between polymeric chains, optimizing the resistance and elasticity of these materials. ${ }^{85}$ Due to their unique combination of physical and chemical properties, chitin/chitosan can be molded relatively easily into porous scaffolds, and their cationic nature allows them to form polyelectrolyte complexes with many types of anionic GAGs, making them capable of modulating the activity of a variety of growth factors and cytokines for tissue-engineering purposes. ${ }^{85}$ An important property of chitin/chitosan is its mucoadhesive nature and its ability to open epithelial tight junctions, making them well-suited for drug delivery across nasal, intestinal, ocular, buccal, and pulmonary systems. ${ }^{85}$ However, some challenges do exist. Chitosan is insoluble in most organic solvents, making delivery of hydrophobic drugs difficult; moreover, various methodologies to adapt the solubilization of chitosan, such as alkylation, acetylation, and carboxymethylation, all come with certain drawbacks and limitations. ${ }^{111}$

Overall, chitin/chitosan is a polymer that will have very important applications in both the industrial and biomedical fields of the future. Its unique chemical properties have recently allowed it to be studied as part of a biological functionalization of microelectromechanical systems, which will enable it to perform functions such as biorecognition, enzymatic catalysis, and controlled drug release, all of which is critical to the advancement of drug-delivery and scaffold technology. ${ }^{85}$

\section{Starch}

Starch is the primary energy reserve polysaccharide in plants, and it is present in the form of granules composed of amylose and amylopectin. ${ }^{112}$ Amylose is a linear polymer composed of glucose monomers linked through $\alpha$-D-(1-4) glycosidic linkages, whereas amylopectin molecules are huge, branched polymers of glucose known to be one of the highest molecular-weight natural polymers. ${ }^{113}$ Different plants have slightly different granule sizes, amylose/amylopectin ratio, mineral contents, and amount of phosphorous and phospholipid contents that lead to varying starch properties. ${ }^{12}$ The specific characterization of starch is particularly important due to different swelling, solubility, gelatinization, mechanical behavior, enzymatic digestibility, rheological characteristics, and surface characteristics, which affect the way it needs to be processed to convert it to a more usable form such as hydrogels, pastes, and nanoparticles. ${ }^{114}$ Generally, the native starch isolated from different plants tends to have limited shear resistance, thermal resistance, thermal decomposition, and a high tendency toward retrogradation. ${ }^{112}$ These limitations have been overcome by combining starch with more stable, synthetic, thermoplastic polymers, or utilizing physical treatment of starch, such as heat or moisture, or utilizing 
chemical modifications, introducing certain functional groups to remarkably alter its physicochemical properties, making starch vastly more useful in tissue engineering, drug delivery, and delivery of biologically active compounds. ${ }^{112}$

Among its tissue-engineering applications, starch is most famous for its usefulness in generating scaffolds for bone regeneration due to its bone-bonding behavior when reinforced with hydroxyapatite, good mechanical properties, non-cytotoxic and biocompatible nature, excellent substrate for cell adhesion, and thermoplastic behavior when combined with thermoplastic polymers. ${ }^{115}$ Lately, further improvements have been made to these starch-based scaffolds. In one recent study, Mahdieh et al synthesized a nanocomposite biomaterial consisting of a blend of thermoplastic starch and ethylene vinyl alcohol as the polymer matrix, and incorporated nano-structured forsterite and vitamin $\mathrm{E}$ as the ceramic reinforcing phase and thermal stabilizer, respectively. ${ }^{116}$ What they found was that nanofosterite, a newly developed bioceramic, resulted in improved biological and mechanical properties, thereby reducing the degradation rate of the scaffold whereas stimulating bone cell proliferation in comparison to the traditional starch-ethylene vinyl alcohol matrix. ${ }^{116}$

In particular, starch and PCL blends (SPCL) have garnered much attention from many research groups for application as a tissue-engineering construct. ${ }^{117}$ PCL improves the processability of starch, reduces its high stiffness, and can overcome the high moisture sensitivity of starch, which is one of the greatest weakness of starch as a biomaterial. ${ }^{118}$ On the other hand, starch improves the biodegradability of PCL and, as the cheapest biomaterial on the planet, starch can substantially lower the high cost of the final product. ${ }^{19}$ Through the appropriate blending of starch and PCL, SPCL can overcome limitations of both PCL and starch, whereas additionally allowing for control over mechanical and degradative properties by adjusting the component ratio - a significant advantage that allows it to conform to the numerous differing tissue-regenerative rates. ${ }^{120}$ SPCL is particularly effective in bone engineering because one of the greatest biological specifications of SPCL composites is their ability to enhance and stimulate osteoblast cell proliferation. ${ }^{117}$ In fact, several different research groups have recently experimented with SPCL for this purpose. ${ }^{117}$ Carvalho et al studied the bone regeneration potential of undifferentiated human adipose-derived stromal/stem cells loaded in SPCL scaffolds for the regeneration of critical-sized mice calvarial defect. ${ }^{121}$ They found that SPCL was a suitable scaffold for bone tissue engineering, allowing for new tissue formation in the calvarial defect to be approximately $20 \%$ of the defect size after 4 weeks and $43 \%$ at 8 weeks. ${ }^{121}$ Link et al did a similar study of critical-sized cranial defects in male Fisher rats and found that the SPCL fiber mesh proved to be an effective osteoconductive material for bone regeneration. ${ }^{122}$ Requicha et al studied the in vivo behavior of a novel, double-layered SPCL scaffold functionalized with silanol groups (SPCL-Si) in a mandibular rodent model and compared the results to a commercial collagen membrane. ${ }^{123}$ They found that SPCL-Si scaffolds induced significantly higher new bone formation when compared to the commercial collagen membrane. ${ }^{123}$

Starch has been proposed as a possible drug-delivery system. ${ }^{124}$ In its hydrogel form, it can efficiently entrap drugs in interstitial spaces, thus protecting them from undesirable conditions in the human body. ${ }^{125}$ In addition, starch hydrogels are resistant to gastric juices, allowing for potential oral drug-delivery systems, and they can be modified to be degraded in very specific portions of the gastrointestinal tract, thus allowing for site-specific delivery. ${ }^{126}$ Moreover, physical modification of starch by retrogradation leads to the development of high levels of type three resistant starch - a very thermally stable, low solubility form of starch that makes it suitable for colon-specific delivery systems. ${ }^{124}$ However, in practice, studies on starch as a possible drug-delivery system have been very limited. Most of the research on starch as part of a drug-delivery system is theoretical, and scientists are still exploring the possible consequences of physical modification of starch on the mechanical and structural properties of hydrogels. To date, there is no conclusive data on this subject.

As the cheapest, most bioavailable, natural polymer, starch is an interesting renewable resource that may have many different biomedical applications. ${ }^{119}$ Starch is entirely biodegradable, noncytotoxic, biocompatible, and exhibits a high Young's modulus with low levels of elongation at break. ${ }^{117}$ It is relatively easy to modify chemically, and it has the ability to replace some more expensive, synthetic polymers in the fabrication of composite biopolymers. ${ }^{127}$ When starch is combined with PCL, SPCL exhibits additional properties, such as better processability, increased mechanical properties, controllable degradability, and enhanced osteoblastic cell proliferation whereas still being relatively cheap to produce. ${ }^{117}$ As the study by Requicha et al showed recently, functionalized SPCL-Si polymers induced much higher rates of bone formation as compared to the traditional (30:70) SPCL blend, further showing the potential of starch-based biopolymers as scaffolds. ${ }^{123}$ However, research outside of bone tissue engineering has largely been limited, and the potential for drug delivery, 
while theoretically studied, has been relatively unexplored as compared to many other natural polymers. Further research needs to be undertaken to exploit the abundance of starch for use in more than just bone tissue engineering.

\section{Alginate}

Alginate is a polysaccharide derived from the cell wall of brown seaweed and extracellularly in some bacteria. It is an anionic polymer that is biocompatible, nontoxic, and noninflammatory - as long as it undergoes multi-purification steps - but it is primarily known for its mild gelation conditions, low cost, and relatively simple modifications in making alginate derivatives with new properties. ${ }^{128}$ In particular, alginate hydrogels have been prepared by various chemical or physical cross-linking methods for diverse applications in wound healing, delivery of bioactive agents, and tissue engineering. ${ }^{129}$ The primary drawbacks of alginate are its generalized lack of strong mechanical properties, poor cell adhesion, and its lack of degradability in mammals. ${ }^{128} \mathrm{How}-$ ever, by combining alginate with other biomaterials such as agarose and chitosan, and by partially oxidizing alginate with molecules like sodium periodate, scientists have managed to enhance its mechanical properties and degradability, conferring significant promise on alginate-based biomaterials. ${ }^{129}$

Lately, there has been a surge of research in the use of alginate for regeneration and engineering of various tissues and organs in the body. One study attempted to create a tissue-engineered skin substitute by developing a fish collagen/alginate (FCA) sponge scaffold that was then functionalized by combining different molecular-weight chitooligosaccharides (COS) with the help of a cross-linking agent. ${ }^{130}$ What they found was that the excellent biological and functional properties of collagen, along with the controllable porosity of sodium alginate, helped create a matrix for the cellular growth in skin tissue regeneration. ${ }^{130}$ The addition of COS to FCA, creating FCA/COS1, resulted in a scaffold with improved cell adhesion and proliferation, ECM compatibility, improved porosity and water uptake, and overall superior physical, mechanical, and biologic properties that could potentially be a candidate for skin tissueengineering application. ${ }^{130}$ Another study blended alginate with poly(ethylene oxide) (PEO), and the resulting product was then modified by acidification of carboxylate groups via trifluoroacetic acid (TFA) to produce poly(alginic acid). ${ }^{131}$ Whereas electrospun scaffolds of sodium alginate are often limited in use for tissue engineering due to high solubility and uncontrollable degradation dynamics, the poly(alginic acid) exhibited an enhanced stability in the aqueous environment and controllable degradability by changing the duration of the TFA-alginate reaction, making it attractive in the production of biomedical devices for tissue engineering. ${ }^{131}$

Alginate - due to its favorable gelation conditions, biocompatibility, and relatively simple modifications - has also been studied for its use in bone regeneration and myocardial tissue regeneration. ${ }^{132}$ One study showed that utilizing alginate as a dispersing agent in hydroxyapatite/chitosan composites helps to create more uniform pore structures than using just hydroxyapatite/chitosan composites. ${ }^{133}$ The increased pore morphology contributed to an increase in the elastic modulus and compressive strength of the scaffold, thus substantially improving osteoblastic differentiation for bone regeneration. ${ }^{133}$ In a separate study, a novel nano-biocomposite scaffold combining chitosan, gelatin, alginate, and hydroxyapatite was shown to have mechanical and biological properties mimicking natural bone. ${ }^{134}$ They found that they could take advantage of alginate's anionic nature, coupled with its excellent cross-linking abilities especially in the presence of multivalent cations - to produce an incredibly stable nanocomposite scaffold with a prolonged degradation time necessary for the formation of neotissue and the ECM. ${ }^{134}$ When it comes to tissue engineering, treatment of cardiac tissue is of notable interest, especially in the case of myocardial infarctions (MIs) where large portions of functional tissue are often lost. In a study of a rat model of acute MI, Kim et al attempted an immediate post-MI local injection of alginate-chitosan hydrogel into the peri-infarct zone and assessed the results 8 weeks later. ${ }^{135}$ What they found was that this treatment promoted greater angiogenesis, increased recruitment of endogenous repair at the infarct zone via recruitment of cardiac stem cells, prevented cell apoptosis, induced cardiomyocyte cell re-entry, and, most importantly, prevented deterioration of cardiac function. ${ }^{135}$ The hydrogelinjected animals demonstrated marked improvements after extensive MI and, given the simplicity of manufacturing and the entirely natural makeup of such a hydrogel, alginatebased biomaterials for myocardial regeneration is likely to play a huge role in cardiovascular repair in the future. ${ }^{135}$ Furthermore, sodium alginate has one of the largest applications in the field of wound healing, due in part to not only its excellent bioresorbable and biocompatible nature, but also because of its ease of gelation and its physical cross-linking abilities, which is often favored over chemical cross-linking due to the ease with which they can be performed. ${ }^{132,136}$ One study formulated freeze-dried wafers combining a 75/25 sodium alginate-to-gelatin ratio and loaded it with silver sulfadiazine (a metal antimicrobial) for application on infected wounds. ${ }^{137}$ 
Sodium alginate gel was used as the main biomaterial primarily because it allows exchange between ions in the wound exudate and the dressing, thereby creating a moist environment that promotes healing. ${ }^{137}$ The gelatin is mixed in to prevent hydrated alginate from losing cation-crosslinkers over time, allowing the wound dressing to release the silver sulfadiazine over a 7-hour period, and severely reducing the bacterial bioburden compared to traditional wound dressings. ${ }^{137}$ Another study found that combining the use of alginate and deoxyribonucleic acid (DNA)-based gels resulted in a sustained release of bioactive factors such as outgrowth endothelial cells, as well as neuropeptides and growth factors for treatment of diabetic foot ulcers, leading to a substantially better healing outcome than the delivery of these bioactive factors alone. ${ }^{138}$ Moreover, in yet a different study, scientists found that integrating hyaluronic acid in an ionically cross-linked alginate matrix hydrogel promoted significant gap closure on dermal wound injuries compared to using either biomaterial alone. ${ }^{139}$ The addition of hyaluronic acid significantly improved the mechanical properties necessary for a wound dressing whereas only very minimally affecting alginate gelation time, providing an effective and easy way to improve excisional wound injuries in a clinical setting. That is not to say that alginate-derived wound dressings have been entirely successful. ${ }^{139}$ In one study, a 500-block randomized prospective study was done to test the effects of a silver-eluting alginate dressing to reduce lowerextremity vascular surgery wound complications compared to a standard dry surgical dressing. They found no significant difference in using this silver alginate dressing in reducing postoperative wound complications. ${ }^{140}$ However, it is clear from these novel studies and others that alginate-based wound dressings will continue to play a significant role in the future as the relative ease of modifiability of alginate properties will allow researchers to explore a limitless number of possibilities and solutions to promote better healing.

Alginate is a widely utilized biomaterial, especially in regenerative medicine and in tissue engineering, due to its biocompatibility, mild and physical gelation process, chemical and physical cross-linking abilities, non-thrombogenic nature, and the resemblance of its hydrogel matrix texture to that of the ECM. ${ }^{132}$ Moreover, alginate happens to be easily modified into any form, such as microspheres, sponges, foams, elastomers, fibers, and hydrogels, thereby broadening the scope of application of alginate-based biomaterials, and it can be combined with other natural biomaterials to create and enhance new and existing properties. ${ }^{141}$ Due to the abundance of algae in water bodies, alginate is one of the most prevalent natural biomaterials in the world, making it relatively a low-cost and feasible biomaterial to use. ${ }^{142}$ However, better control of polymer properties and development of its tissue-interactive forms are necessary for breakthroughs in many tissue-engineering applications. ${ }^{132}$ The introduction of cell-interactive features to alginate biomaterials will become crucial in the future to properly produce replacement tissues and even organs. ${ }^{132}$ The type of adhesion ligands and spatial organizations in the hydrogel of alginates are key for proper functioning of regenerated tissues, and although arginylglycylaspartic acid (RGD) peptides have been used mostly to date as a cell adhesion ligand, multiple ligands in combination - along with solubility factors - will be necessary to further our application of alginate-based biomaterials for regenerative purposes. ${ }^{129}$ For wound-healing applications, alginate-based gels will need to play a more active role, incorporating one or more bioactive agents to facilitate wound healing as compared to the rather passive process they play in current clinical applications. ${ }^{129}$ The future of alginate-based wound dressings hinges upon establishing more control over the delivery of one or more drugs, as well as their duration and sequence of release while considering external environmental changes. ${ }^{129}$ Furthering our understanding of the fundamentals of alginate properties will help researchers take advantage of the remarkable properties and bioavailability of alginate and utilize genetic engineering techniques to control the bacterial synthesis of alginate with new and improved properties, thus revolutionizing the use of this material. ${ }^{129}$

\section{Silk}

Silk fibers are natural biopolymers derived primarily from the silkworm Bombyx mori. ${ }^{143}$ The silk fiber consists of two parallel SF proteins, held together by a layer of silk sericin protein glue on the surface. ${ }^{144}$ Until recently, silk sericin has been deemed to be immunologically incompatible with the human body and has, therefore, been largely neglected as a biopolymer. ${ }^{145}$ However, SF has been used as a biomedical suture material for centuries. ${ }^{143}$ It is a semi-crystalline structure that has an incredible combination of mechanical properties, possessing very high tensile strength, coupled with excellent elasticity and flexibility. ${ }^{143}$ In fact, its strengthto-density ratio is up to ten times higher than that of steel. ${ }^{146}$ SF's unique mechanical properties, tunable biodegradability, diverse side-chain chemistries, and the fact that genetic engineering techniques can be used to tailor the protein, allows it to have a variety of novel properties, functions, and applications in the biomedical field. ${ }^{143}$ Furthermore, SF derived from other species in the order Lepidoptera often have other unique 
properties, increasing their potential for biomedical use. ${ }^{145}$ In addition, some spiders, such as Antheraea mylitta, have been found to produce silks that possess better cell adhesion and a more highly ordered crystalline structure, leading to increased mechanical strength and lower solubility in acidic solvents. ${ }^{145}$ Additionally, because SF is easily processed into gels, films, nanoparticles, membranes, nanofibers, scaffolds, and foam-like forms, they can be adapted to mimic a tremendous diversity of tissues in the human body. ${ }^{143}$ As a result, SF has lately been studied for use in several different applications, including almost all fields of tissue engineering, wound repair, drug delivery, and even as a possible bio-ink for 3D bioprinting. ${ }^{147}$

SF's unique combination of elasticity, strength, and potential self-healing modifications (via cross-linking), along with its biocompatibility, tunable biodegradation, anti-bacterial, and other mechanical properties, makes it an attractive material to include as a part of a composite scaffold for tissue engineering. ${ }^{148}$ Several different studies in the past few years have explored SF-based biomaterials for tissue engineering. In one study, Shao et al designed a nanostructured composite scaffold with the core consisting of hydroxyapatite and SF. ${ }^{149}$ The composite fibers were fabricated by electrospinning $-\mathrm{a}$ technique used to generate nanoscale polymeric fibers using electrical energy - to create a nanofiber composite scaffold, and then subsequently compared to nanofibers of pure SF. ${ }^{149}$ They found that the composite scaffold demonstrated a 90-fold and 2-fold increase in initial modulus and breaking stress, respectively. ${ }^{149}$ Osteoblast-like cells were cultivated on the composite, and Shao et al found that the composite scaffold demonstrated increased biocompatibility, better cell adhesion, and proliferation as well as functionally promoted alkaline phosphatase and biomineralization. ${ }^{149}$ The group concluded that the nanostructured composite scaffold consisting of a hydroxyapatite and SF core has excellent biomimetic and mechanical properties and has potential as a biocompatible scaffold for bone tissue engineering. ${ }^{149}$ In another study, Tian et al utilized the same coaxial electrospinning technique to fabricate a nanofiber scaffold ( $\mathrm{p}-\mathrm{PS} / \mathrm{N}$ ) consisting of nerve growth factor, SF, and PLA. ${ }^{150}$ After 11 days, the PC12 cells (a model for neuronal differentiation) that were cultured on these scaffolds showed elongated neurites with lengths up to $95 \mu \mathrm{m}$, leading the research group to conclude that $\mathrm{p}-\mathrm{PS} / \mathrm{N}$ scaffolds were able to support the attachment and differentiation of PC12 cells for nerve tissue engineering. ${ }^{150}$ Another research group independently developed a $3 \mathrm{D}$ porous $\mathrm{SF}$ scaffold derived from the non-mulberry muga silkworm of Antheraea assamensis to examine its ability to support cartilage tissue engineering. ${ }^{151}$ They found that the SF scaffold could generate enhanced sulfated glycosaminoglycans and type II collagen, and demonstrated in vivo biocompatibility after 8 weeks of implantation in a subcutaneous model of rat, suggesting that these non-mulberry SF scaffold may be suitable for chondrocyte-based cartilage repair. ${ }^{151}$ Moreover, in a separate study, that same research group developed a 3D-blended scaffold consisting of SF and human hair-derived keratin to investigate their ability to promote enhanced fibroblast cell adhesion and proliferation. ${ }^{152}$ They found that the scaffold demonstrated high porosity and interconnected pores, with excellent thermal, degradation, and mechanical properties. ${ }^{152}$ In addition, they found increased expression of collagen type I in cultured cells, demonstrating functional fibroblast proliferation, and the research group concluded that blended biomaterials, specifically SF and human hair keratin-blended scaffolds, may have a promising future as a dermal substitute for skin tissue engineering. ${ }^{152}$ In an attempt to engender enthesis (functional repair of tendons and ligaments), another research group, Tellado et al, engineered a complex scaffold consisting of biphasic SF scaffolds with integrated anisotropic and isotropic pore alignment, similar to what is found in native tendons/ligaments and bone/ cartilage, respectively. ${ }^{153}$ The scaffolds were functionalized with heparin, and human primary adipose-derived mesenchymal stem cells were cultured on the scaffold and assessed for their ability to deliver transforming growth factor $\beta 2$ (TGF- $\beta 2$ ) and growth/differentiation factor 5 (GDF5). ${ }^{153}$ This research group found that heparin functionalization increased the amount of TGF- $\beta 2$ and GDF5 attached to the scaffold, leading to enhanced expression of cartilage and collagen II protein contents and enthesis, demonstrating that growth factor-loaded biphasic SF scaffolds may be useful in tendon/ligament repair. ${ }^{153}$

In addition to its numerous tissue-engineering applications, SF has recently been explored for its use as a biomaterial for skin repair due to its excellent hemostatic properties, low inflammatory potential, and permeability to oxygen and water vapor. ${ }^{154}$ Preliminary studies have even shown that SF film and sponge-based dressings promote wound healing and enhance skin regeneration compared to traditional hydrocolloids. ${ }^{154,155}$ Currently to date, there are only three SF-based medical products approved for clinical use in the world: SeriScaffold (Allergan Medical, Inc.) from the US FDA, TymPaSil (CG Bio Inc.) from the Ministry of Food and Drug Safety of South Korea and Sidaiyi (Suzhou Soho Biomaterial Science and Technology Co., Ltd) from the China Food and Drug Administration. ${ }^{156}$ Of these three, 
only Sidaiyi is indicated for skin wound healing, but none of these three products are widely utilized in clinical practice. ${ }^{156}$ A very recent clinical study done in 2017 by Zhang et al focused on developing a translational SF film for clinical application. ${ }^{156}$ They conducted a single-blind, parallel, controlled clinical trial on 71 patients for treating donor-site wounds. ${ }^{156}$ Compared to the positive control Sidaiyi, the SF film demonstrated substantially faster wound healing, taking approximately 9.86 days on average to complete wound healing, compared to 11.35 days for Sidaiyi. ${ }^{156}$ Further, $100 \%$ of the patients treated with the SF film healed by Day 14, whereas $88.6 \%$ of patients treated with Sidaiyi healed by Day $19 .{ }^{156}$ Only one inflammatory reaction case and zero adverse reaction cases was noticed in the SF film group, whereas three inflammatory reaction cases and four adverse cases were recorded in the Sidaiyi group. ${ }^{156}$ The SF film group had cleaner wounds compared to the exudate-prone wounds of the Sidaiyi group, due to its better fluid handling capacity and gas permeability. ${ }^{156}$ This translational study, taken together with its previously successful studies on rabbit and porcine wound models, demonstrates that this newly developed SF film may indeed be safer and more effective than the Sidaiyi material currently available for clinical use in China for the treatment of skin repair and regeneration. ${ }^{156}$ To date, many other studies have recently shown positive in vitro results with electrospun nanofiber SF dressings, or composite SF dressings, as a way to enhance the bulk properties of SF. ${ }^{157-161}$ Based on all these studies, it is clear that SF potentially has a very good outlook for the future. ${ }^{156}$

Besides the properties already mentioned, SF has many other unique and standout properties that make it excellent for use as a drug carrier. ${ }^{148}$ It can allow for loading of even the most sensitive of drugs, such as proteins and nucleic acids, due to its mild, all-aqueous processing conditions. ${ }^{148}$ Moreover, SF has a diverse range of amino acids with several functional groups that can simplify the attachment of different types of biomolecules or antibodies, giving it a wide degree of functionalization. ${ }^{162}$ Finally, SF naturally has an intrinsic respond to $\mathrm{pH}$ changes, making it easy to control drug-release kinetics, and the mechanism of elimination from the body can easily be done by degradation via proteolytic enzymes in the body, leaving no likely side effects. ${ }^{163}$ Recently, most research groups have been investigating the mechanistic component of incorporating SF nanoparticulate for protein delivery, small-molecules delivery, and even anticancer delivery. ${ }^{164-166}$ These studies have been investigating properties of SF such as electrostatic interactions for loading efficiency, different drug-delivery mediums for controlling drug-release kinetics, and encapsulation efficiency, as well as compatibility, degradability, and drug retention. ${ }^{163}$ However, SF's potential role in drug delivery has yet to be established due to the relative infancy of SF nanoparticulation, and further intensive examinations regarding SF properties and how to best exploit and improve those properties for drug delivery remains to be seen. ${ }^{163}$

Tissue-engineering techniques at present, while having improved vastly from years before, still fail to capture the complexity of the 3D anatomy and functionality of human tissues and, as a result, very few engineered constructs reach human clinical trials. ${ }^{147} 3 \mathrm{D}$ bioprinting offers an untapped potential to capture the complexity of human tissues, and it has been touted as the future of tissue-regeneration strategies. ${ }^{167}$ One of the most crucial aspects of 3D printing is bio-ink design, which not only provides the $3 \mathrm{D}$ architecture but also acts as the first point of contact for cells to synthesize regulatory proteins and cytokines appropriate for the tissue it is mimicking. ${ }^{168}$ Silk has become one of the most popular choices for bio-ink preparations. The ability to physically cross-link its protein polymer chains via inter- and intramolecular $\beta$-sheet semi-crystalline structure formation allows it to be stabilized after printing without the need for any chemical or photochemical reactions or additives. ${ }^{169}$ In addition, silk is a very strong and robust material as previously mentioned, and its inherent spinnability, cytocompatibility, and controllable degradability make silk a very strong candidate for future bio-ink preparations. ${ }^{147}$ Of course, studies are still in its infancy stages and, so far, reported literature on silk $3 \mathrm{D}$ bioprinting has demonstrated cell viability after printing to be anywhere from $45 \%$ to $98 \% .{ }^{170}$ However, of the limited available hydrogel bio-inks currently tested, optimized blends of B. Mori silk-gelatin bio-inks has shown the most potential for 3D bioprinting of functional tissue equivalents. ${ }^{147}$ Significant scientific and regulatory challenges still remain before $3 \mathrm{D}$ bioprinting technology can hit commercialization levels, and more experimental studies need to be done on optimizing silk as a potential bio-ink. ${ }^{147}$

SF-based biomaterials have immense potential as one of the preeminent natural biopolymers studied today. Its ease of structural modification, controllable degradability, high tensile strength, elasticity and flexibility, potential to introduce physical cross-links, hemostatic and self-healing attributes, and its ability to be processed into numerous different forms, such as sponges, films, and hydrogels, make SF a polymer with various biomedical applications. ${ }^{143}$ However, unlike starch or more bioavailable polymers, silk is produced by only a select number of species, and only in B. Mori are 
they available in appreciable amounts. ${ }^{143}$ Although spider silk has been shown to display impressive toughness, stiffness, strength, and extensibility, it is impractical to obtain any large quantities of silk from any spider. ${ }^{145}$ One study has pointed to the transgenic expression of spider silk in plants (eg, tobacco and potato) or utilizing mammalian epithelial cells as a way to garner a more substantive production of silk, but so far, no solutions are in place. ${ }^{171,172}$ Besides sustainability, the key to developing new silk-incorporated biomaterials and to advance our current technology in tissue engineering is to work on surface modifications or compositing with other synthetic polymers and to figure out how to control crystallization in SF when it is thermally treated or mechanically stretched. ${ }^{145}$ With novel cross-linking methods, SF-based materials can be designed to self-heal, which will result in new applications for tissue engineering and wound healing. ${ }^{145}$ Silk sericin - the neglected protein in silk fibers - also needs to be studied in more depth. While it has long been deemed biologically incompatible with the human body, new studies show that silk sericin is only immunogenic when associated in conjunction with SF. ${ }^{145}$ When used by itself, or combined with other biopolymers, sericin has been shown to have attractive bioactive properties, with its antioxidant character, moisturizing ability, and mitogenic effect on mammalian cells. ${ }^{173}$ Its promotion of keratinocytes and fibroblasts have led to the development of sericin-based biomaterials for skin tissue repair, and its ability to be cross-linked with genipin can allow for it to be used in bone, dermal, and neural tissue engineering. ${ }^{174}$ Furthermore, sericin may be utilized for drug delivery because it can help facilitate the fabrication of nanoand microparticles, hydrogels, and conjugated molecules, through its chemical reactivity and $\mathrm{pH}$-responsiveness, thus improving the bioactivity of drugs. ${ }^{173}$ Currently, methods of purifying silk sericin protein have been mostly met with unpredictable results in size, composition, and biological activity, but future technological advancements and recovery methods may lead silk sericin to be an important biomaterial in the field of tissue engineering and drug delivery. ${ }^{144}$

\section{Polyhydroxyalkanoates (PHA)}

PHA is a class of natural, biodegradable polyesters synthesized by microorganisms as intracellular carbon- and energystorage compounds in uneven growth conditions. ${ }^{175}$ They have exceptional biodegradability and biocompatibility, and produce nontoxic degradation products, making them excellent for use in biomedical applications such as drug delivery, tissue engineering, and substitute for implantable devices, ${ }^{176}$ including sutures, repair patches slings, orthopedic pins,

\section{Poly-(R)-3-hydroxyalkanoate}

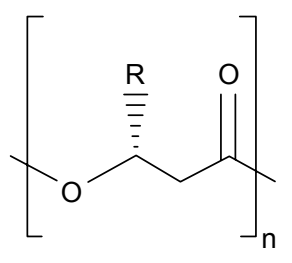

Poly-(R)-3-hydroxybutyrate

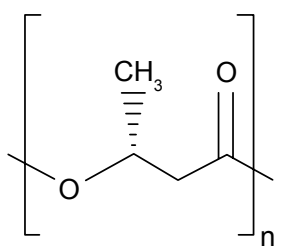

Figure 3 Structure of poly-(R)-hydroxybutyrate (polyhydroxyalkanoate, PHA).

scaffold, stents, and adhesion barriers. ${ }^{177}$ However, unmodified PHAs - despite their exceptional abilities mentioned above - have some important limitations, such as the presence of large crystals causing poor mechanical properties, ${ }^{178}$ poor thermal stability, high hydrophobicity, and slow degradation rate, rendering them unfavorable to be used for many biomedical applications. ${ }^{179}$ The intrinsic hydrophobicity of PHAs impedes their utilization in biomedical applications, ${ }^{177}$ as many biomedical appliances require better hydrophilicity. ${ }^{179}$ Moreover, PHAs are lacking in chemical functionalities, and the polyesters are frequently incompatible when combined with drugs. ${ }^{177}$ Although biodegradable, PHAs are extremely stable when unmodified, thus diminishing their therapeutic functions in other areas. ${ }^{177}$ Meanwhile, uncommon PHAs possessing functional side groups including hydroxyl- and/or carboxyl groups, methylated-branches, and other hydrophilic derivatives have been produced by a few organisms to broaden the applicability of PHAs. ${ }^{180}$ Therefore, it is crucial that PHAs need to be modified to fix these properties while maintaining their exceptional characteristics to make them capable for use in biomedical applications.

A very important character of PHAs is that they can be developed in a way to have various physicochemical behaviors in properties such as amphiphilicity, crystallinity, and mechanical properties by simply choosing an appropriate production strain, cultivation conditions, and carbon sources. ${ }^{181,182}$ Because PHAs are generated by over 300 different types of Gram-positive and Gram-negative bacteria, there are numerous ways to produce different properties of PHAs, making PHA the largest group of biopolymers and, therefore, one of the most promising in biomedical applications. ${ }^{176}$ In addition to the biosynthesis of PHA using 
different bacteria, there are several alternative approaches that have been developed, making PHA a vastly accessible biopolymer. Blending one PHA with another PHA, blending PHAs with other biodegradable polymers and polyesters, and chemical modifications of PHAs such as grafting and copolymerization and, recently, electrospinning, ${ }^{183}$ all allow easy, and more importantly, precise modulation of the PHA structure, leading to predictable functionalities. ${ }^{179}$ Whereas blending has been shown to produce properties in PHAs that fall within extremes, grafting and copolymerization have led to a development of a variety of PHAs with different characteristics that may be used for several biomedical applications. ${ }^{179}$ With the advantages gained from the development of metabolic engineering, PHAs with various combinations of monomers with differing proportions can be also produced from inexpensive substrates, such as glucose and fatty acids. ${ }^{175}$

PHAs have been widely studied for a vast field of biomaterial applications. For example, one study compared the various properties of different PHA scaffolds and their fabrication techniques for use in bone tissue engineering and posited that composite scaffolds via mixing different PHA materials may produce even better scaffolds. ${ }^{184}$ Another study specifically identified poly-3-hydroxyoctanoate - a type of PHA generated by Pseudomonas mendocina - as a PHA biomaterial that successfully showed great, distinctive properties for cardiac tissue engineering. ${ }^{185}$ A different study studied solution-cast films and nonwoven electrospun membranes prepared from poly(3-hydroxybutyrate-co-4hydroxybutyrate) solutions as experimental wound dressings and found that they accelerated wound vascularization and the healing process. ${ }^{186}$ Another study assessed a different variation of PHA - a composite scaffold consisting of PHA/ ceramic composites - which showed greater bioactivity and bone regenerating potential both in vitro and in vivo. ${ }^{187}$

A couple of studies found that, compared to either poly(3hydroxybutyrate) (PHB) or poly(ethylene glycol) (PEG), a novel alternating block copolymer based on $\mathrm{PHB}$ and $\mathrm{PEG}$ (PHB-alt-PEG) had enhanced tunable mechanical properties and improved processability while remaining non-cytotoxic and thereby overcoming significant limitations mentioned previously of single unmodified PHAs. ${ }^{188}$ Furthermore, Loh et al found that, by adjusting the 3-hydroxybutyrate (3HB) to ethylene glycol ratio in this synthesized thermogelling PHBalt-PEG copolymer or the concentration of the copolymer in the hydrogel itself, the drug-release rates of multi-block PHB-alt-PEG can be easily controlled. ${ }^{189}$ Further study has shown that the PHB-alt-PEG system can also be used as a long-term drug-delivery system for a mouse model of hepatocellular carcinoma by changing the concentration of the gel, showing significant potential for further development in anticancer applications. ${ }^{190}$

In addition, within the last few years, discoveries regarding more therapeutic applications of PHA monomers have emerged, including the treatment of epilepsy and neurodegenerative disorders. A study showed that increasing the blood concentration of $3 \mathrm{HB}$ - the monomer of $\mathrm{PHB}$ - was useful in controlling seizures: the mean latency to the onset of seizure was significantly prolonged in 3HB-treated mice, suggesting that the ketone body $3 \mathrm{HB}$ maintains potential as an anticonvulsant for patients with epilepsy. ${ }^{191}$ Another study investigating murine L929 fibroblasts, human umbilical vein endothelial cells, and rabbit articular cartilages demonstrated that $3 \mathrm{HB}$ had a stimulatory effect on cell-cycle progression that was mediated by a signaling pathway dependent upon increases in $\left[\mathrm{Ca}^{2+}\right] .{ }^{192}$ Similarly, Maalouf et al found that $3 \mathrm{HB}$ significantly decreased the mitochondrial production of ROS and the accompanying excitotoxic changes by increasing nicotinamide adenine dinucleotide (NADH) oxidation in the mitochondrial respiratory chain, but did not affect levels of the endogenous antioxidant glutathione. Therefore, $3 \mathrm{HB}$ reduced glutamate-induced free radical formation by improving the $\mathrm{NAD}^{+} / \mathrm{NADH}$ ratio and increasing mitochondrial respiration in neocortical neurons. ${ }^{193}$ In the same study, 3 HB in combination with acetoacetate reduced neuronal cell death and prohibited changes in neuronal membrane properties. Together, these studies indicate that the ability to modify and design PHAs with a wide range of accessible properties shows great promise for PHAs as potential therapeutic biomaterials.

Clearly, extensive research has already been done on PHAs and their potential for success, but there has only been one FDA-approved PHA for biomedical use - poly(4hydroxybutyrate) - which has very high elasticity for use as an absorbable suture. ${ }^{194}$ Because each individually modified PHA needs to undergo extensive tests to prove that biocompatibility and biodegradability are still as exceptional as it is in its unmodified form, more tests need to be done for PHA use to be expanded. This is particularly true of composite PHAs, which seem to have nearly unlimited potential in creating diverse biomaterials with even better properties for their specific purpose. Furthermore, despite their enormous potential, commercial uptake of PHAs is limited due to the inconsistent polymer properties and high production costs of the raw polymer. ${ }^{195}$ Moreover, sustainability is another 
significant issue. One recent article advocated that because substrates for PHA production can be found in carbon-rich waste located mostly in economically poor countries, integration of biopolymer production into these areas will provide both sustainable PHA production and a more extensive labor market for these countries. ${ }^{196}$ However, in order for such sustainable practice to happen, policymakers, scientists, and the heads of the relevant industrial branches will need to work together.

\section{Sundew adhesives}

Sundew adhesive is a natural polysaccharide-based hydrogel which was discovered when scientists began to investigate a carnivorous plant known for its peculiar lifestyle. ${ }^{197}$ This plant, the Sundew Drosera, uniquely utilizes two different glands: the sessile gland, which secretes digestive enzymes, and the stalked gland, which produces a sticky exudate to attract and then restrict prey from escaping. ${ }^{197}$ This sticky exudate produced by the latter gland has, in particular, garnered the interest of many researchers due to not only its biocompatibility, biodegradability, and eco-friendly nature, but also because of its antibiotic characteristics, its unique properties of enhancing cell adhesion and differentiation, and for its extremely high elasticity. ${ }^{198}$ In fact, its elasticity has been shown in studies to be so remarkable that it can be drawn into threads of approximately $1 \mathrm{~m}$ in length. ${ }^{198}$ Upon further investigation, scientists have discovered the chemical structure of the exudate produced by Drosera to be a bioadhesive formed by xylose, mannose, galactose, glucuronic acid, and ester sulfate in the ratio of $1: 6: 6: 6: 1 .{ }^{199}$

Studies done on the Sundew have mostly returned with positive findings. For example, Zhang et al found that drying the adhesive produced by the sundew plant allowed neuronlike cells to attach and grow on the nanofibers made by the dried adhesive, showing the potential of the sundew adhesive for tissue engineering. ${ }^{199}$ In more recent studies, fibrous scaffolds obtained from the Sundew adhesive have been found to increase adhesion of numerous types of cells, including fibroblast and smooth muscle cells. It was found that nanonetworks within the sundew adhesives exhibit viscoelastic behaviors that allow for, among other things, more powerful adhesion of multiple mammalian cells. ${ }^{197}$

However, despite the promise Sundew adhesives have shown, some concerns have been raised that may limit the potential of the sundew adhesives. One study found that the sundew adhesives are highly susceptible to temperature variation and that adhesion strength was substantially diminished at temperatures between $-20^{\circ} \mathrm{C}$ and $-80^{\circ} \mathrm{C}$. ${ }^{197}$ However, a much more important issue that may overshadow the sundew adhesives' therapeutic potential is the ability to collect sufficient quantities of the hydrogel. ${ }^{200}$ However, Sun et al were able to develop a sundew-inspired adhesive hydrogel mimicking the native sundew adhesives with superior adhesive strength, nanostructure, and resistance to shearing compared to other hydrogels in vitro, whereas also demonstrating superior wound-healing capabilities when paired with mouse adipose-derived stem cells in vivo. ${ }^{200}$ Despite these findings, much more study needs to be done to see if there are more efficient methods of collecting the natural Sundew adhesive, and to assess the safety and efficacy of sundew-inspired adhesive hydrogels.

\section{Ivy nanoparticles}

Ivy, which belongs to the genus Hedera, is known for the unique ability to affix itself to and grow upwards on surfaces such as rocks, trees, and fences, to name just a few. ${ }^{201}$ To do this, ivy has been found to use adhering disks generated by the stem of the Ivy plant. ${ }^{201}$ These adhering disks consist of four to seven tendrils or "fingers" which generate a substance called ivy nanoparticles (INPs). ${ }^{201}$ These INPs are entirely organic and are formed by a group of macromolecules composed of arabinogalactan proteins, and it is the INPs that are primarily responsible for the immense amount of force the Ivy can generate. ${ }^{202}$ In fact, it has been shown that an ivy disc weighing just $0.5 \mathrm{mg}$ can produce approximately $0.9 \mathrm{~kg}$ pull-off force, which is 1.8 million times larger than the weight of the adhering disc itself. ${ }^{203}$ This incredible adhesive strength - combined with their excellent aqueous solubility, low intrinsic viscosity, biocompatibility, and biodegradability - demonstrate the immense potential of INPs in the field of tissue engineering. ${ }^{14}$

In one recent study investigating the potential of INPs in drug delivery, INP-conjugated doxorubicin exhibited a stronger cytotoxic effect against multiple cancer cell lines in vitro and in vivo. ${ }^{14}$ In that same study, INPs were also found to allow stronger adhesion of smooth muscle cells in the collagen scaffolds once they were embedded with INPs, showing that not only can INPs be used as drug carriers for cancer therapy, but also as nano-fillers to enrich scaffolds. ${ }^{14}$ In addition, INPs demonstrate unique optical properties that exhibit strong Ultraviolet (UV) absorbance and scattering, potentially allowing them to play an important role as a sunscreen protective agent. ${ }^{204}$ Currently, most sunscreens today utilize metal oxide nanoparticles as fillers; however, 
environmental concerns, as well as safety concerns of using these nanoparticles on skin, make using traditional sunscreens less than ideal. When compared to $\mathrm{TiO}_{2}$ nanoparticles, which are the metal oxide nanoparticles used frequently in traditional sunscreens today, the ivy nanoparticles block more UV light, are less toxic to mammalian cells, and are more easily biodegradable, showing that ivy nanoparticles may potentially be used as a better, safer sunscreen agent. ${ }^{205}$

Considering their excellent physical profile, it is likely there are far more applications for these INPs. Unfortunately, application of INPs is severely limited due to the obscurity they have been in. ${ }^{202}$ In fact, the structure of INPs has only recently been identified. More studies need to be done to verify their safety and efficacy, as well as to discover a sustainable way to produce these nanoparticles - whether they are naturally derived or synthetically mimicked. ${ }^{202}$

\section{Synthetic biodegradable polymers}

Although natural polymers have demonstrated the potential advantage of supporting cell function and adhesion, there are a few limitations and concerns with regard to their use. For example, it is difficult to control the mechanical properties and degradation rates of natural polymers, and there exists the potential for a natural polymer to elicit an immune response or carry microbes or viruses. ${ }^{16,206}$ In contrast, synthetic polymers can be modified to possess a much wider range of mechanical and chemical properties than natural polymers. Although synthetic polymers can avoid problems with immunogenicity, biocompatibility poses a new challenge. ${ }^{207}$ Thus, degradable synthetic polymers are currently being studied extensively in order to avoid the potential long-term effects associated with non-degradable polymers, such as scarring and inflammation. ${ }^{207,208}$ Meanwhile, synthetic polymers can be produced under controlled conditions and thus exhibit predictable and reproducible mechanical and physical properties such as tensile strength, elastic modulus, and degradation rate. ${ }^{207}$ A further advantage of synthetic polymers is the control of material impurities. Lastly, pure synthetic polymers with well-defined and simple structures have lower possible risks with regard to toxicity, immunogenicity, and infections.

\section{Saturated aliphatic polyesters}

Saturated aliphatic polyesters, such as poly(glycolic acid) (PGA), PLA, and PLGA copolymers, are the most often used biodegradable synthetic polymers for $3 \mathrm{D}$ scaffolds in tissue engineering. ${ }^{207-211}$ The chemical properties of these polymers allow hydrolytic degradation through de-esterification. For example, PLA and PGA can be processed easily, and their degradation rates and physical and mechanical properties are adjustable over a wide range by using various molecular weights, structure, composition, and copolymers. ${ }^{207,208,211}$ Moreover, the body contains highly regulated mechanisms for completely removing monomeric components of glycolic and lactic acids when these polyesters degrade: glycolic acid is converted to metabolites or eliminated by other mechanisms, whereas lactic acid can be cleared through the tricarboxylic acid cycle. ${ }^{212}$ Due to their biodegradability and biocompatibility, PGA and PLA have been approved by the FDA for use in medical devices, such as degradable sutures and other implantable devices. ${ }^{213}$

\section{PGA}

PGA is a hydrophilic and highly crystalline polymer with a relatively fast degradation rate. It degrades rapidly in aqueous solutions or in vivo, and loses its mechanical integrity between 2 and 4 weeks - depending on the molecular weight and the degradation conditions. ${ }^{212,214}$ Moreover, PGA has great flexibility in the tuning of its material properties and physical parameters such as pore size and tortuosity, which are important for developing scaffold-based tissue-engineering constructs. ${ }^{212,214}$ Thus, PGA can be used not only in tissue engineering, but also in wastewater treatment, food products, and other biomedical applications such as drug delivery or biological glues. ${ }^{215-219}$ Furthermore, previous studies have successfully developed PGA sheets combined with fibrin glue to treat open soft tissue wounds during oral surgery. ${ }^{220,221}$ Similarly, PGA sheets with fibrin glue were efficient at prohibiting postoperative bleeding, reducing postoperative pain, and enhancing epithelialization during the reconstruction of bone surfaces following tumor resection in the oral cavity. ${ }^{222}$ However, the relatively fast degradation rate - along with the acidic degradation products and low solubility confer inherent disadvantages and, consequently, limit the biomedical applications for PGA. ${ }^{223}$ Thus, ongoing research

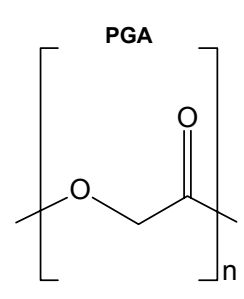

Figure 4 Structure of poly(glycolic acid) (PGA). 
continues with regard to several PGA-based copolymers in order to bypass these obstacles.

\section{PLA}

PLA is another widely used biodegradable scaffolding material in biomedical applications. ${ }^{224}$ Although similar in structure to PGA, PLA exhibits different chemical, physical, and mechanical properties due to the presence of an extra methyl group in its repeating units. For example, it can take months to years to lose the mechanical integrity of a PLA scaffold, ${ }^{224}$ which makes PLA a more suitable biomaterial for loadbearing applications, such as orthopedic fixation devices. To date, multiple PLA-based orthopedic products are available in the market, including the Phantom Soft Thread Soft Tissue Fixation Screw ${ }^{\circledR}$ (DePuy), Phantom Suture Anchor ${ }^{\circledR}$ (DePuy), Full Thread Bio Interference Screw ${ }^{\circledR}$ (Arthrex), BioScrew $^{\circledR}$ (Conmed), Bio-Anchor ${ }^{\circledR}$ (Conmed), Meniscal Stinger $^{\circledR}$ (Linvatec), and the Clearfix Meniscal Dart ${ }^{\circledR}$ (Innovasive Devices). A novel promising biomedical engineering application for PLA involves photoluminescent graphene quantum dots (GQDs) with a large surface area and superior mechanical flexibility, which possess interesting optical and electronic properties. ${ }^{225}$ A recent study reported that the multifunctional nanocomposite of PLA and PEG-grafted GQDs (f-GQDs) is biocompatible with low cytotoxicity, which makes it suitable for simultaneous intracellular microRNAs (miRNAs) imaging analysis as well as gene delivery. ${ }^{225}$ These results underscore the potential of the extremely versatile multifunctional nanocomposite - f-GQDs - in biomedical applications of intracellular molecular analysis and clinical gene therapeutics.

Three forms of PLA exist: ${ }_{L}$-PLA, ${ }_{D}-P L A$, and a racemic mixture of ${ }_{D, L}$-PLA. Recently, the stereoisomer ${ }_{D, L}$-PLA has been significantly explored as a biomedical coating for orthopedic material due to its high mechanical stability and excellent biocompatibility. ${ }^{207,226,227}$ Furthermore, ${ }_{D, L}$-PLA of low molecular weight can be combined with drugs such as growth factors, antibiotics, or thrombin inhibitors to establish a locally acting drug-delivery system. ${ }^{9}$ Thus, recent efforts have shifted focus toward applying ${ }_{\mathrm{D}, \mathrm{L}}$-PLA as a scaffold material for tissue engineering due to these highly desirable features.

\section{PLGA}

PLGA with varying lactide/glycolide ratios can be synthesized to achieve intermediate degradation rates between PLA and PGA. Generally, the copolymer PLGA is favored in comparison with its constituent homopolymers for the development of bone substitute constructs because PLGA offers greater control of degradation properties by varying the ratio between its monomers. ${ }^{228}$ For example, PLGA possesses a wide range of degradation rates dictated by the composition of chains, hydrophobic/hydrophilic balance, and crystallinity. ${ }^{228}$ However, despite biocompatibility, the clinical application of pure PLGA for bone regeneration is hindered by low osteoinductivity and suboptimal mechanical properties for load-bearing applications. Thus, PLGA is typically used in conjunction with other materials, such as ceramics or bioactive glass, and is routinely modified in order to render it more biomimetic, thereby improving its ability to enhance bone regeneration. ${ }^{229}$ By implanting AgNP/PLGA composite grafts into grossly infected critical-sized bone segmental defects, our group demonstrated that AgNP/PLGA composite grafts possess significant antibacterial properties and osteoconductivity in vivo. ${ }^{209}$ In a follow-up experiment, we unexpectedly found that AgNP/PLGA-coated stainless steel alloy materials not only exhibited strong antibacterial activity but also presented significant osteoinductivity that was not observed in their individual components alone. ${ }^{210}$ Similarly, Shi et al reported that PLGA/hydroxyapatite microsphere composites with the bisphosphonate-based osteoporosis-preventing drug alendronate significantly inhibited the growth of macrophages, which have been identified as precursors of osteoclasts and as being potentially responsible for osteoporosis, while improving osteoblast proliferation and upregulating the expression of a key osteogenic enzyme - alkaline phosphatase. ${ }^{230}$ Together, these findings require more research into the osteoconductive and osteoinductive properties of PLGA-based systems
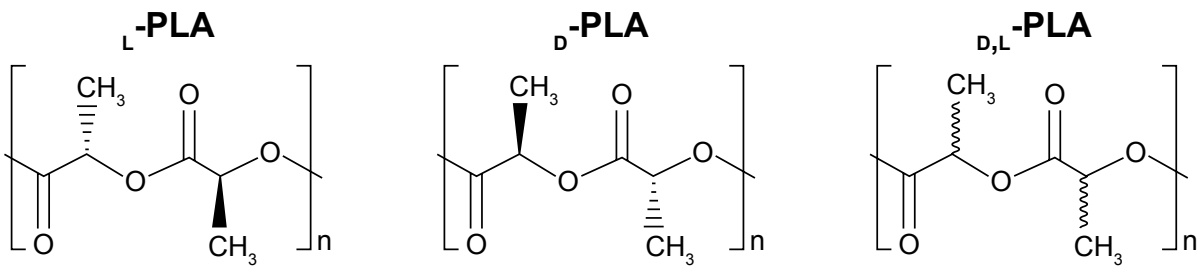

Figure 5 Structure of poly(lactic acid) isomers (L-PLA, D-PLA, D,L-PLA) 


\section{PLGA}

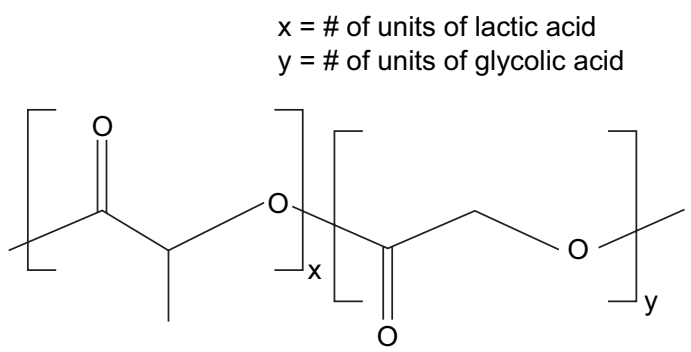

Figure 6 Structure of poly(lactic acid-co-glycolic acid) (PLGA). X=number of units of lactic acid and $Y=$ number of units of glycolic acid.

but, nevertheless, offer promising therapeutic material for orthopedic surgery.

\section{Poly(E-caprolactone) (PCL)}

PCL is another saturated aliphatic biodegradable polyester used in the development of tissue-engineering scaffolds and other biomedical applications. ${ }^{231-233}$ PCL is a semi-crystalline polymer with a melting temperature of $55^{\circ} \mathrm{C}-60^{\circ} \mathrm{C}$ and a very low glass-transition temperature of approximately $-54^{\circ} \mathrm{C}$ and, thus, it tends to maintain a rubbery state and high material permeability under physiological conditions. ${ }^{231-233}$ It can be degraded by microorganism, hydrolytic, enzymatic, or intracellular mechanisms under physiological conditions; however, PCL's slow degradation rate of 2-4 years, compared to PLA, PGA, and PLGA, along with its hydrophobicity makes it less attractive for general tissue-regeneration applications and more attractive for long-term implants and drug-delivery systems. ${ }^{234,235}$ A recent study demonstrated that gravity-spun collagen-coated PCL fibers enhanced proliferation rates of human osteoblast cells. ${ }^{234}$ Accordingly, these findings underscore the potential of gravity-spun PCL fibers as a delivery platform for ECM proteins to enhance cell adherence and proliferation for tissue repair. Similarly, PCL has been used to effectively entrap antibiotic drugs and, subsequently, was incorporated into a drug-delivery system for enhancing bone ingrowth and regeneration in the treatment of bone defects. ${ }^{236}$ Moreover, ongoing research continues regarding micro- and nanoscale drug-delivery vehicle systems centered on PCL. ${ }^{237}$

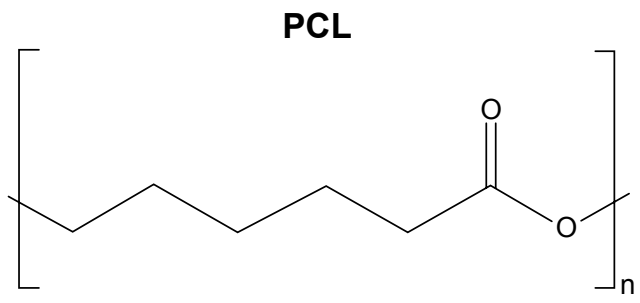

Figure 7 Structure of poly(e-caprolactone) (PCL).
Additionally, a solid, freeform, fabrication-based injection molding process has been developed for the fabrication of PCL, and the resulting PCL-calcium phosphate scaffolds display in vitro cytocompatibility and suitable mechanical properties for hard tissue repair. ${ }^{238}$ Further, Chiari et al have reported the viability of using a composite matrix composed of PCL and HA as a possible meniscus substitute. ${ }^{239}$ Overall, PCL is a useful biodegradable biomaterial deserving further investigation as a porous scaffold for bone tissue engineering and as a drug-delivery vehicle.

\section{Polyanhydrides}

PLA, PGA, and PLGA possess immense utility for drug delivery, but they do have limitations, including a tendency for a non-uniform release profile with certain drugs. Thus, in response to these problems, polyanhydrides were created. Polyanhydrides were initially designed for drug-delivery applications due to their hydrophobicity and ability to undergo degradation through surface erosion rather than bulk degradation, which allows for a constant release profile for certain drugs and is especially important in the case of extremely potent drugs. ${ }^{240}$ Polyanhydrides are biocompatible and degradable in vivo into nontoxic diacid byproducts, which are eliminated from the body as metabolites. ${ }^{240}$ For these reasons, drugs can be well protected when implanted in such polymers due to the fact that almost no water penetrates before the polymer erodes. ${ }^{241}$ Therefore, in 1996, polyanhydrides were approved as a drug-delivery vehicle by the FDA following comprehensive drug release and biocompatibility evaluations. ${ }^{242}$

Generally, polyanhydrides can be easily synthesized from widely available, low-cost sources and have been modified to possess desirable characteristics. ${ }^{243}$ Poly[(carboxyphenoxy propane)-(sebacic acid)] is the most widely investigated FDA-approved polyanhydride. It is typically utilized as a localized delivery vehicle, specifically for the controlled delivery of the chemotherapeutic agent bis-chloroethyl nitrosourea during brain cancer treatment (Gliadel ${ }^{\circledR}$, Arbor Pharmaceuticals, LLC). ${ }^{244}$ Moreover, the results of previous

\section{Polyanhydride}

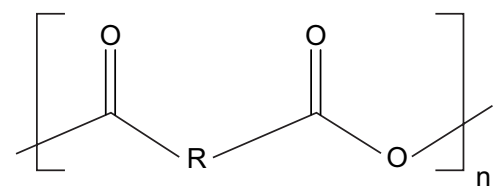

Figure 8 Structure of polyanhydride. 
animal studies were validated in a recent human trial, verifying the promising potential of this polymer drug-delivery system. ${ }^{244}$ Clearly, future exploration of polyanhydrides in drug-delivery systems is warranted.

\section{Polyurethane (PUR)}

Owing to their toughness, durability, biocompatibility, and biostability, PURs are a favorable choice for medical devices and, since the 1960s, they have been utilized typically as biostable and inert materials in heart valves, vascular grafts, catheters, and prostheses. ${ }^{245,246}$ However, in the late 1990s, interest regarding the design of biodegradable PURs for tissue engineering and drug delivery surged due to their relative sensitivity to biodegradation as well as the desire to further understand the biological mechanisms for in vivo biodegradation. ${ }^{246}$ Biodegradable PURs possess convincing potential as scaffolds for tissue regeneration. ${ }^{245,246}$ They exhibit a broad range of versatility in terms of modifiable mechanical properties, biological properties, physical properties, biodegradability, and blood and tissue compatibility due to their segmented-block structural character.

PURs are normally synthesized through a polycondensation reaction of diisocyanates with alcohols/amines, whereas the synthesis of biodegradable PURs allows for the incorporation of hydrolyzable segments into their backbone. ${ }^{247}$ Nevertheless, the toxicity of common diisocyanates such as toluene diisocyanate and $4,4^{\prime}$-methylenediphenyl diisocyanate has led to the consideration of other biocompatible aliphatic diisocyanates for developing a new generation of biodegradable PURs. Recently, the development of biocompatible aliphatic diisocyanates and amino acid-derived diisocyanates possessing reduced toxicity, such as lysine diisocyanate and 1,4-diisocyanatobutane, has allowed for novel opportunities to synthesize biocompatible and biodegradable PURs that can enhance cell proliferation and adhesion without adverse effects. ${ }^{247}$ Moreover, a biodegradable elastic PUR - Degrapol ${ }^{\circledR}$ (Abmedica) - is currently being used to develop a highly porous scaffold for tissueengineering applications. ${ }^{248}$ It is also worth noting that a unique, injectable, two-component lysine-diisocyanatebased PUR system - PolyNova ${ }^{\circledR}$ (PolyNovo Biomaterials Pvt. Ltd) - has been developed for orthopedic applications. ${ }^{249}$ PolyNova ${ }^{\circledR}$ polymerizes at physiological temperature in situ and, thus, arthroscopic administration in its liquid form results in suitable mechanical support and comparable or superior bonding strength relative to standard bone cement. Additionally, it supports favorable cell adhesion and proliferation. ${ }^{249}$
Together, the research indicates the promising potential for PURs in various biomedical applications, such as porous scaffolds for tissue engineering and drug delivery. ${ }^{250}$

\section{Polyphosphazenes}

Polyphosphazenes are a relatively newer class of inorganicorganic hybrid polymers composed of an inorganic backbone of repeating phosphorus and nitrogen atoms with alternating single and double bonds. To date, polyphosphazenes have been investigated as potential biodegradable biomaterials due to their synthetic flexibility, unparalleled functionality, and adaptability for numerous applications.

The phosphorous-nitrogen backbone of polyphosphazenes confers extraordinary flexibility, whereas their side groups determine the different properties of these polymers. Considering these features, polymers possessing highly controlled properties including solubility, hydrophobicity/ hydrophilicity, extent of crystallinity, and appropriate thermal transitions can be designed and developed through modification of their side groups. ${ }^{251}$ Furthermore, the degradation profiles of the polymers can be controlled by modifying their side groups to achieve appropriate degradation profiles ranging from a few hours to years. ${ }^{251}$ Laurencin et al studied different poly[(amino acid ester) phosphazenes] and revealed that polyphosphazenes showed the fastest degradation after modification with glycine ethyl ester. ${ }^{252}$ In contrast with polyesters, the poly[(amino acid ester) phosphazenes] degrade into neutral and nontoxic products such as ammonia, phosphates, and the corresponding ester side groups. ${ }^{252}$ Taking advantage of this unique property, a recent study combined polyphosphazenes with PLGA to form self-neutralizing blend systems. ${ }^{253}$ In addition, polyphosphazenes are suitable for drug delivery with their distinct ability to undergo both surface and bulk erosion while maintaining controllable rates and modes of degradation. ${ }^{254}$

\section{Polyphosphazene}

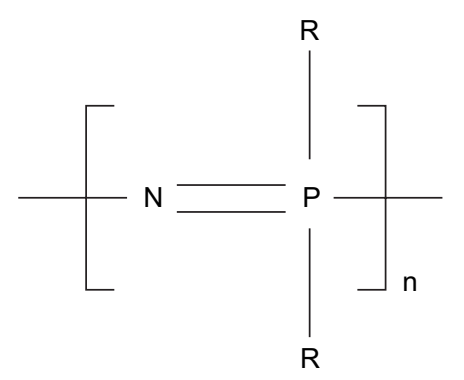

Figure 9 Structure of polyphosphazene. 
With regard to biocompatibility and toxicity, the majority of the poly[(amino acid ester) phosphazenes] implanted subcutaneously elicited minimal to mild tissue responses. ${ }^{251,255-257}$ Furthermore, several of the poly[(amino acid ester) phosphazenes] have demonstrated significant osteoconductivity and have been explored as matrices for bone tissue engineering. ${ }^{251,255}$ Recently, by utilizing the advantageous interactions between polyphosphazene side groups and calcium phosphate ceramics, a polyphosphazene-self setting calcium phosphate composite cement system was created. ${ }^{258}$ However, as a result of the flexible backbone, many of the poly[(amino acid ester) phosphazenes] are soft elastomeric polymers and, thus, present limitations as a biomaterial used for load-bearing applications. Additionally, a recent study found success in using a glycine-based photo-polymerizable polyphosphazene as a scaffold for adipose tissue regeneration. ${ }^{259}$ Preliminary results demonstrated the non-cytotoxic nature of the polymers and their degradation products and the cell adhesion and proliferation of adipose-derived stem cells. ${ }^{259}$ Meanwhile, another report suggests that blends with PLGA may benefit the application of polyphosphazenes in osteogenesis. The initial hydrolytic degradation of PLGA generates a porous structure with some residual strength, which would then degrade over a much longer period of time. Therefore, fine-tuning the system could potentially yield biomaterials with tissue-engineering properties superior to those of PLGA. ${ }^{260}$ However, their utility as scaffolds in tissue engineering is still under investigation and warrants further studies.

In truth, with regard to on-the-market applications and clinical studies, polyphosphazenes are severely lacking compared to the more established polymers for drug-delivery applications. However, the many promising in vitro and in vivo studies spanning a broad range of therapies highlights the potential of polyphosphazenes in this area. ${ }^{251-260}$ Furthermore, the inherent high functionality of the phosphorus-nitrogen backbone along with the intrinsic, tunable, biodegradability of polyphosphazenes ${ }^{251}$ underscores their immense potential as a group of biomaterials for drug delivery and other biomedical applications.

\section{Conclusion}

Due to the presence of a wide diversity of biomaterials, both natural and synthetic, varying quality of material formulation, and a general lack of comparative studies of different biomaterials for specific biomedical applications, it is impossible to conclude which polymer is the most ideal. In general, natural biomaterials have greater inherent biocompatibility compared to synthetic biomaterials, but they are also mechanically inferior, as their mechanical, structural, and chemical properties cannot be altered in the same way as synthetic biomaterials.

However, instead of trying to categorize specific polymers for specific biomedical applications, it seems that the future of biopolymer application is to utilize different combinations of polymers to develop hybrid polymers that have a better specificity profile for specific biomedical applications - be it for tissue engineering, drug delivery, or wound healing. Indeed, numerous recent studies regarding polymers for biomedical use have addressed combining different biomaterials, via techniques like blending, grafting, and chemical cross-linking reactions, and the results have been mostly positive. With techniques like electrospinning which allows for the creation of various different forms of nanoscale polymers, such as nanotubes, nanofibers, and nanospheres - combined with the nearly limitless potential of generating numerous different combinations of natural and synthetic biomaterials, there exists a very good chance that more effective biomaterial-based materials can be fabricated with the proper biocompatibility, degradation, and physicochemical properties for a specific biomedical application. Numerous challenges still exist today across most or all biomaterials, such as the feasibility of mass production at a relatively low cost as well as overcoming certain physiochemical limitations of specific biomaterials; however, with the advent of cloning and genetic engineering techniques to express both native and synthetic biomaterials in a variety of host systems, there remains a bright future for biopolymeric use in medicine.

Currently, in the field of tissue engineering, temporary, artificial composite scaffolds are being researched and developed for cells to adhere to, differentiate, and form new tissue. However, there is an increased interest in developing $3 \mathrm{D}$ scaffolds that can not only support tissue regeneration, but also act as a biomatrix that can support cues and signals to promote functional tissue connections. ${ }^{145}$ One increasingly popular way to generate such a scaffold is to utilize 3D bioprinting techniques, incorporating polymeric biomaterials as the bio-ink. This technology will allow us to have more control in the scaffold properties that were traditionally very difficult or impossible to control, such as cell distribution, fluid flow, and porosity. These resulting 3D-engineered constructs will allow us to mimic the properties of tissue in the human body better than any other traditionally engineered approach, making it more desirable for tissue regeneration. The ultimate goal of 3D bioprinting is to eventually be able to develop patient-specific tissues and organs, and although several technical, scientific, regulatory, and even 
ethical challenges still exist, there has been an exponential increase in research and interest into making such a technology a reality.

Drug-delivery systems traditionally are either orally administered or injected, but problems exist in using such methods for protein and nucleic acid delivery. Furthermore, traditional drug-delivery systems still face problems with drug side effects, efficacy, and patient compliance. Recently, the use of nanotechnology has ushered in a novel strategy for drug delivery. Utilizing nanotechnology has led to the development of numerous novel carriers that are capable of releasing not only a wider range of molecules, proteins, peptides, and nucleic acids, but also allows for a more specific targeted delivery with controlled release. Nanoparticles based on biodegradable and biocompatible polymers, in particular, have recently shown potential in cancer therapy and as sustained drug-delivery vehicles, prolonging drug half-life, improving solubility, and reducing immunogenicity. Furthermore, they have shown the ability to simultaneously co-deliver multiple drugs, making patient compliance more likely whereas also enhancing potential synergistic effects of certain drugs and suppressing drug resistance. These biodegradable and biocompatible polymeric-based nanoparticulation can also be applied in wound-healing applications via delivery of bioactive agents. Current clinical wound-healing applications play a much more passive role, and development of this technology will help with better, more efficient wound healing. Nanosized particles for drug delivery clearly demonstrate significant advantages over traditional drug-delivery systems, and the key to future development of drug-delivery systems is the increased understanding and application of nanotechnology.

\section{Disclosure}

The authors report no conflicts of interest in this work.

\section{References}

1. Piskin E. Biodegradable polymers as biomaterials. J Biomater Sci Polym Ed. 1995;6(9):775-795.

2. Taylor P. Global Markets For Implantable Biomaterials [market report on the Internet]. Massachusetts: BCC Research. 2015 Jan [cited March 1, 2016]. Available from: http://www.bccresearch.com/market-research/ advanced-materials/implantable-biomaterials-markets-report-avm118a. html. Accessed February, 2018.

3. Barbucci R. Integrated Biomaterials Science. New York: Kluwer Academic/Plenum Publishers; 2002.

4. Langer R, Vacanti JP. Tissue engineering. Science (New York, N. Y.). 1993;260(5110):920-926.

5. Vacanti JP, Langer R. Tissue engineering: the design and fabrication of living replacement devices for surgical reconstruction and transplantation. Lancet. 1999;354(Suppl 1):S32-S34.

6. Dvir T, Timko BP, Kohane DS, Langer R. Nanotechnological strategies for engineering complex tissues. Nat Nanotechnol. 2011;6(1):13-22.
7. Kohane DS, Langer R. Polymeric Biomaterials in Tissue Engineering. Pediatr Res. 2008;63(5):487-491.

8. Vert M. Aliphatic Polyesters: Great Degradable Polymers That Cannot Do Everything. Biomacromolecules. 2005;6(2):538-546.

9. Gollwitzer H, Ibrahim K, Meyer H, Mittelmeier W, Busch R, Stemberger A. Antibacterial poly(D,L-lactic acid) coating of medical implants using a biodegradable drug delivery technology. J Antimicrob Chemother. 2003;51(3):585-591.

10. Freyman TM, Yannas IV, Yokoo R, Gibson LJ. Fibroblast contraction of a collagen-GAG matrix. Biomaterials. 2001;22(21):2883-2891.

11. Marijnissen WJCM, van Osch GJVM, Aigner J, et al. Alginate as a chondrocyte-delivery substance in combination with a non-woven scaffold for cartilage tissue engineering. Biomaterials. 2002;23(6):1511-1517.

12. Park H, Choi B, Hu J, Lee M. Injectable chitosan hyaluronic acid hydrogels for cartilage tissue engineering. Acta Biomater. 2013;9(1): $4779-4786$.

13. Lenaghan SC, Serpersu K, Xia L, He W, Zhang M. A naturally occurring nanomaterial from the Sundew (Drosera) for tissue engineering. Bioinspir Biomim. 2011;6(4):046009.

14. Huang Y, Wang YJ, Wang Y, et al. Exploring naturally occurring ivy nanoparticles as an alternative biomaterial. Acta Biomater. 2015;25: 268-283.

15. Gelse K, Pöschl E, Aigner T, Structure C-. function, and biosynthesis. Advanced Drug Delivery Reviews. 2003;55(12):1531-1546.

16. Lee $\mathrm{CH}$, Singla A, Lee Y. Biomedical applications of collagen. Int $J$ Pharm. 2001;221(1-2):1-22.

17. Chattopadhyay S, Raines RT. Review collagen-based biomaterials for wound healing. Biopolymers. 2014;101(8):821-833.

18. Kretlow JD, Young S, Klouda L, Wong M, Mikos AG. Injectable Biomaterials for Regenerating Complex Craniofacial Tissues. Adv Mater (Deerfield Beach, Fla). 2009;21(32-33):3368-3393.

19. Pomahač B, Svensjö T, Yao F, Brown H, Eriksson E. Tissue Engineering of Skin. Crit Rev Oral Biol Med. 1998;9(3):333-344.

20. Vin F, Teot L, Meaume S. The healing properties of Promogran in venous leg ulcers. $J$ Wound Care. 2002;11(9):335-341.

21. Awad HA, Boivin GP, Dressler MR, Smith FNL, Young RG, Butler DL. Repair of patellar tendon injuries using a cell-collagen composite. J Orthop Res. 2003;21(3):420-431.

22. Juncosa-Melvin N, Shearn JT, Boivin GP, et al. Effects of Mechanical Stimulation on the Biomechanics and Histology of Stem Cell-Collagen Sponge Constructs for Rabbit Patellar Tendon Repair. Tissue Eng. 2006; 12(8):2291-2300.

23. Juncosa-Melvin N, Matlin KS, Holdcraft RW, Nirmalanandhan VS, Butler DL. Mechanical Stimulation Increases Collagen Type I and Collagen Type III Gene Expression of Stem Cell-Collagen Sponge Constructs for Patellar Tendon Repair. Tissue Eng. 2007;13(6):1219-1226.

24. Atala A. Tissue engineering for bladder substitution. World J Urol. 2000;18(5):364-370.

25. Liu S, Peulve $\mathrm{P}$, Jin O, et al. Axonal regrowth through collagen tubes bridging the spinal cord to nerve roots. J Neurosci Res. 1997;49(4): 425-432.

26. Bozkurt A, Claeys KG, Schrading S, et al. Clinical and biometrical 12-month follow-up in patients after reconstruction of the sural nerve biopsy defect by the collagen-based nerve guide Neuromaix. Eur $J$ Med Res. 2017;22(1):34.

27. Narotam PK, José S, Nathoo N, Taylon C, Vora Y, Matrix C. Collagen Matrix (DuraGen) in Dural Repair: Analysis of a New Modified Technique. Spine. 2004;29(24):2868-28669.

28. Gruessner U, Clemens M, Pahlplatz PV, Sperling P, Witte J, Rosen HR. Improvement of perineal wound healing by local administration of gentamicin-impregnated collagen fleeces after abdominoperineal excision of rectal cancer. Am J Surg. 2001;182(5):502-509.

29. John P, Lazarus F, George JP, Selvam A, Prabhuji ML. Adjunctive Effects of A Piscean Collagen-Based Controlled-Release Chlorhexidine Chip in the Treatment of Chronic Periodontitis: A Clinical and Microbiological Study. J Clin Diagn Res. 2015;9(5):ZC70-ZC74.

30. Geiger M, Rh L, Friess W. Collagen sponges for bone regeneration with rhBMP-2. Adv Drug Deliv Rev. 2003;55(12):1613-1629. 
31. Jovanovic SA, Hunt DR, Bernard GW, Spiekermann H, Wozney JM, Wikesjö UME. Bone reconstruction following implantation of rhBMP-2 and guided bone regeneration in canine alveolar ridge defects. Clin Oral Implants Res. 2007;18(2):224-230.

32. Sano A, Maeda M, Nagahara S, et al. Atelocollagen for protein and gene delivery. Adv Drug Deliv Rev. 2003;55(12):1651-1677.

33. Koide T. Designed triple-helical peptides as tools for collagen biochemistry and matrix engineering. Philosophical Trans Royal Society B Biol Sci. 2007;362(1484):1281-1291.

34. Badylak SF, Gilbert TW. Immune response to biologic scaffold materials. Semin Immunol. 2008;20(2):109-116.

35. An B, Kaplan DL, Brodsky B. Engineered recombinant bacterial collagen as an alternative collagen-based biomaterial for tissue engineering. Front Chem. 2014;2:40.

36. Smit NW, Ten Sande JN, Parvizi M, et al. Recombinant human collagenbased microspheres mitigate cardiac conduction slowing induced by adipose tissue-derived stromal cells. PLoS One. 2017;12(8):e0183481.

37. Tang Y, Yang X, Hang B, et al. Efficient Production of Hydroxylated Human-Like Collagen Via the Co-Expression of Three Key Genes in Escherichia coli Origami (DE3). Appl Biochem Biotechnol. 2016;178(7): 1458-1470.

38. Peng YY, Howell L, Stoichevska V, Werkmeister JA, Dumsday GJ, Ramshaw JAM. Towards scalable production of a collagen-like protein from Streptococcus pyogenes for biomedical applications. Microb Cell Fact. 2012;11(1):146-146.

39. Brodsky B, Ramshaw JA. Bioengineered Collagens. In: Parry DAD, Squire JM, editors. Fibrous Proteins: Structures and Mechanisms. Cham: Springer International Publishing; 2017:601-629.

40. Ghasemi-Mobarakeh L, Prabhakaran MP, Morshed M, NasrEsfahani M-H, Ramakrishna S, Poly E. Electrospun poly( $\varepsilon$-caprolactone)/ gelatin nanofibrous scaffolds for nerve tissue engineering. Biomaterials. 2008;29(34):4532-4539.

41. Burke CJ, Hsu TA, Formulation VDB. stability, and delivery of live attenuated vaccines for human use. Crit Rev Ther Drug Carrier Syst. 1999; 16(1):1-83.

42. Gou Y, Miao D, Zhou M, Wang L, Zhou H, Su G. Bio-Inspired ProteinBased Nanoformulations for Cancer Theranostics. Front Pharmacol. 2018;9:421.

43. Saddler JM, Horsey PJ. The new generation gelatins. A review of their history, manufacture and properties. Anaesthesia. 1987;42(9):998-1004.

44. Foox M, Zilberman M. Drug delivery from gelatin-based systems. Expert Opin Drug Deliv. 2015;12(9):1547-1563.

45. Ozkizilcik A, Tuzlakoglu K. A new method for the production of gelatin microparticles for controlled protein release from porous polymeric scaffolds. J Tissue Eng Regen Med. 2014;8(3):242-247.

46. Lakshminarayanan R, Sridhar R, Loh XJ, et al. Interaction of gelatin with polyenes modulates antifungal activity and biocompatibility of electrospun fiber mats. Int J Nanomedicine. 2014;9:2439-2458.

47. Cohen B, Shefy-Peleg A, Zilberman M. Novel gelatin/alginate soft tissue adhesives loaded with drugs for pain management: structure and properties. J Biomater Sci Polym Ed. 2014;25(3):224-240.

48. Karim AA, Bhat R. Fish gelatin: properties, challenges, and prospects as an alternative to mammalian gelatins. Food Hydrocoll. 2009;23(3): 563-576.

49. Bigi A, Cojazzi G, Panzavolta S, Roveri N, Rubini K. Stabilization of gelatin films by crosslinking with genipin. Biomaterials. 2002;23(24): 4827-4832.

50. Rajangam T, Ssa A. Fibrinogen and fibrin based micro and nano scaffolds incorporated with drugs, proteins, cells and genes for therapeutic biomedical applications. Int J Nanomedicine. 2013;8:3641-3662.

51. Ahmed TAE, Dare EV, Hincke M. Fibrin: A Versatile Scaffold for Tissue Engineering Applications. Tissue Eng Part B Rev. 2008;14(2): 199-215.

52. Kjaergard HK, Velada JL, Pedersen JH, Fleron H, Hollingsbee DA. Comparative Kinetics of Polymerisation of Three Fibrin Sealants and Influence on Timing of Tissue Adhesion. Thromb Res. 2000;98(2): $221-228$.
53. Tredwell SJ, Sawatzky B. The Use of Fibrin Sealant to Reduce Blood Loss During Cotrel-Dubousset Instrumentation for Idiopathic Scoliosis. Spine. 1990;15(9):913-915.

54. Mcgill V, Kowal-Vern A, Lee M, et al. Use of Fibrin Sealant in Thermal Injury. J Burn Care Rehabil. 1997;18(5):429-434.

55. Jackson MR, Macphee MJ, Drohan WN, Alving BM. Fibrin sealant: Current and potential clinical applications. Blood Coagul Fibrinolysis. 1996;7(8):737-746.

56. Briganti E, Spiller D, Mirtelli C, et al. A composite fibrin-based scaffold for controlled delivery of bioactive pro-angiogenetic growth factors. $J$ Control Release. 2010;142(1):14-21.

57. Barsotti MC, Magera A, Armani C, et al. Fibrin acts as biomimetic niche inducing both differentiation and stem cell marker expression of early human endothelial progenitor cells. Cell Prolif. 2011;44(1):33-48.

58. de La Puente P, Ludeña D, López M, Ramos J, Iglesias J. Differentiation within autologous fibrin scaffolds of porcine dermal cells with the mesenchymal stem cell phenotype. Exp Cell Res. 2013;319(3):144-152.

59. Zisch AH, Schenk U, Schense JC, Sakiyama-Elbert SE, Hubbell JA. Covalently conjugated VEGF-fibrin matrices for endothelialization. J Control Release. 2001;72(1-3):101-113.

60. de La Puente P, Ludeña D. Cell culture in autologous fibrin scaffolds for applications in tissue engineering. Exp Cell Res. 2014;322(1):1-11.

61. Pradeep AR, Nagpal K, Karvekar S, Patnaik K, Naik SB, Guruprasad CN. Platelet-Rich Fibrin With 1\% Metformin for the Treatment of Intrabony Defects in Chronic Periodontitis: A Randomized Controlled Clinical Trial. J Periodontol. 2015;86(6):729-737.

62. Ajwani H, Shetty S, Gopalakrishnan D, et al. Comparative evaluation of platelet-rich fibrin biomaterial and open flap debridement in the treatment of two and three wall intrabony defects. $J$ Int Oral Health. 2015;7(4):32-37.

63. Wong C, Inman E, Spaethe R, Helgerson S. Fibrin-based biomaterials to deliver human growth factors. Thromb Haemost. 2003;89(3): 573-582.

64. de La Puente P, Ludeña D, Fernández A, Aranda JL, Varela G, Iglesias J. Autologous fibrin scaffolds cultured dermal fibroblasts and enriched with encapsulated bFGF for tissue engineering. J Biomed Mater Res A. 2011;99A(4):648-654.

65. Linnes MP, Ratner BD, Giachelli CM. A fibrinogen-based precision microporous scaffold for tissue engineering. Biomaterials. 2007;28(35): $5298-5306$

66. Toole BP. Hyaluronan: from extracellular glue to pericellular cue. Nat Rev Cancer. 2004;4(7):528-539.

67. El Maradny E, Kanayama N, Kobayashi H, et al. The role of hyaluronic acid as a mediator and regulator of cervical ripening. Hum Reprod. 1997;12(5):1080-1088.

68. Meyer IK, Palmer JW. The polysaccharides of the vitreous humor. J Biol Chem. 1934;107:629-634.

69. Mori M, Yamaguchi M, Sumitomo S, Takai Y. Hyaluronan-based Biomaterials in Tissue Engineering. Acta Histochem Cytochem. 2004; 37(1):1-5.

70. Allison DD, Grande-Allen KJ, Review G-AKJ. Review. Hyaluronan: A Powerful Tissue Engineering Tool. Tissue Eng. 2006;12(8): 2131-2140.

71. Prestwich GD. Engineering a clinically-useful matrix for cell therapy. Organogenesis. 2008;4(1):42-47.

72. Burdick JA, Prestwich GD. Hyaluronic Acid Hydrogels for Biomedical Applications. Adv Mater. 2011;23(12):H41-H56.

73. Yu A, Niiyama H, Kondo S, Yamamoto A, Suzuki R, Kuroyanagi Y. Wound dressing composed of hyaluronic acid and collagen containing EGF or bFGF: comparative culture study. $J$ Biomater Sci Polym Ed. 2013;24(8):1015-1026.

74. Niiyama H, Kuroyanagi Y. Development of novel wound dressing composed of hyaluronic acid and collagen sponge containing epidermal growth factor and vitamin C derivative. J ArtifOrgans. 2014;17(1):81-87.

75. Prestwich GD. Hyaluronic acid-based clinical biomaterials derived for cell and molecule delivery in regenerative medicine. J Control Release. 2011;155(2):193-199. 
76. Hunt DR, Jovanovic SA, Wikesjö UME, Wozney JM, Bernard GW. Hyaluronan Supports Recombinant Human Bone Morphogenetic Protein-2 Induced Bone Reconstruction of Advanced Alveolar Ridge Defects in Dogs. A Pilot Study. J Periodontol. 2001;72(5):651-658.

77. Eppley BL, Dadvand B. Injectable Soft-Tissue Fillers: Clinical Overview. Plast Reconstr Surg. 2006;118(4):98e-106e.

78. Yoo HS, Lee EA, Yoon JJ, Park TG. Hyaluronic acid modified biodegradable scaffolds for cartilage tissue engineering. Biomaterials. 2005; 26(14):1925-1933.

79. Kato Y, Nakamura S, Nishimura M. Beneficial actions of hyaluronan (HA) on arthritic joints: effects of molecular weight of HA on elasticity of cartilage matrix. Biorheology. 2006;43(3-4):347-354.

80. Lepidi S, Grego F, Vindigni V, et al. Hyaluronan Biodegradable Scaffold for Small-caliber Artery Grafting: Preliminary Results in an Animal Model. Euro J Vasc Endovasc Surg. 2006;32(4):411-417.

81. Kumar MNVR, Muzzarelli RAA, Muzzarelli C, Sashiwa H, Domb AJ. Chitosan Chemistry and Pharmaceutical Perspectives. Chem Rev. 2004;104(12):6017-6084.

82. Raveendran S, Rochani A, Maekawa T, Kumar D. Smart Carriers and Nanohealers: A Nanomedical Insight on Natural Polymers. Materials. 2017;10(8):929.

83. Muzzarelli RAA. Human enzymatic activities related to the therapeutic administration of chitin derivatives. Cell Mol Life Sci. 1997;53(2) 131-140.

84. Kim IY, Seo SJ, Moon HS, et al. Chitosan and its derivatives for tissue engineering applications. Biotechnol Adv. 2008;26(1):1-21.

85. Ahsan SM, Thomas M, Reddy KK, Sooraparaju SG, Asthana A, Bhatnagar I. Chitosan as biomaterial in drug delivery and tissue engineering. Int J Biol Macromol. 2017.

86. Chung Y-C, Chen C-Y. Antibacterial characteristics and activity of acid-soluble chitosan. Bioresour Technol. 2008;99(8):2806-2814

87. Akman AC, Tigli RS, Gumusderelioglu M, Nohutcu RM. bFGF-loaded HA-chitosan: a promising scaffold for periodontal tissue engineering. J Biomed Mater Res A. 2010;92(3):953-962.

88. Gobin AS, Butler CE, Mathur AB. Repair and Regeneration of the Abdominal Wall Musculofascial Defect Using Silk Fibroin-Chitosan Blend. Tissue Eng. 2006;12(12):3383-3394.

89. Wu X, Black L, Santacana-Laffitte G, Patrick CW. Preparation and assessment of glutaraldehyde-crosslinked collagen-chitosan hydrogels for adipose tissue engineering. J Biomed Mater Res A. 2007;81A(1): $59-65$.

90. Sun T, Khan TH, Sultana N. Fabrication and In Vitro Evaluation of Nanosized Hydroxyapatite/Chitosan-Based Tissue Engineering Scaffolds. J Nanomater. 2014;2014(1):1-8.

91. Levengood SL, Zhang M. Chitosan-based scaffolds for bone tissue engineering. J Mater Chem B. 2014;2(21):3161-3184.

92. Cicco SR, Vona D, deGiglio E, et al. Chemically Modified Diatoms Biosilica for Bone Cell Growth with Combined Drug-Delivery and Antioxidant Properties. Chempluschem. 2015;80(7):1104-1112.

93. Tamburaci S, Tihminlioglu F. Diatomite reinforced chitosan composite membrane as potential scaffold for guided bone regeneration. Materials Sci Eng C. 2017;80:222-231.

94. di Martino A, Sittinger M, Risbud MV. Chitosan: A versatile biopolymer for orthopaedic tissue-engineering. Biomaterials. 2005;26(30): 5983-5990.

95. Dodane V, Vilivalam VD. Pharmaceutical applications of chitosan. Pharm Sci Technolo Today. 1998;1(6):246-253.

96. Madhumathi K, Sudheesh Kumar PT, Abhilash S, et al. Development of novel chitin/nanosilver composite scaffolds for wound dressing applications. J Mater Sci Mater Med. 2010;21(2):807-813.

97. Kumar PTS, Abhilash S, Manzoor K, Nair SV, Tamura H, Jayakumar R. Preparation and characterization of novel $\beta$-chitin/nanosilver composite scaffolds for wound dressing applications. Carbohydr Polym. 2010;80(3):761-767.

98. Dev A, Mohan JC, Sreeja V, et al. Novel carboxymethyl chitin nanoparticles for cancer drug delivery applications. Carbohydr Polym. 2010;79(4):1073-1079.
99. Gu J, Al-Bayati K, Ho EA, Ea H. Development of antibody-modified chitosan nanoparticles for the targeted delivery of siRNA across the blood-brain barrier as a strategy for inhibiting HIV replication in astrocytes. Drug Deliv Transl Res. 2017;7(4):497-506.

100. Ramana LN, Sharma S, Sethuraman S, Ranga U, Krishnan UM. Evaluation of chitosan nanoformulations as potent anti-HIV therapeutic systems. Biochim Biophys Acta. 1840;2014(1):476-484.

101. Dev A, Binulal NS, Anitha A, et al. Preparation of poly(lactic acid)/ chitosan nanoparticles for anti-HIV drug delivery applications. Carbohydrate Polymers. 2010;80(3):833-838.

102. Wu J, Jiang W, Shen Y, Jiang W, Tian R. Synthesis and characterization of mesoporous magnetic nanocomposites wrapped with chitosan gatekeepers for $\mathrm{pH}$-sensitive controlled release of doxorubicin. Mater Sci Eng C Mater Biol Appl. 2017;70(Pt 1):132-140.

103. di J, Yu J, Wang Q, et al. Ultrasound-triggered noninvasive regulation of blood glucose levels using microgels integrated with insulin nanocapsules. Nano Research. 2017;10(4):1393-1402.

104. Liang J, Yan H, Puligundla P, Gao X, Zhou Y, Wan X. Applications of chitosan nanoparticles to enhance absorption and bioavailability of tea polyphenols: A review. Food Hydrocolloids. 2017;69: 286-292.

105. Jing Z-W, Ma Z-W, Li C, et al. Chitosan cross-linked with poly (ethylene glycol)dialdehyde via reductive amination as effective controlled release carriers for oral protein drug delivery. Bioorg Med Chem Lett. 2017;27(4):1003-1006.

106. Qiao X, Peng X, Qiao J, et al. Evaluation of a photocrosslinkable hydroxyethyl chitosan hydrogel as a potential drug release system for glaucoma surgery. J Mater Sci Mater Med. 2017;28(10):149.

107. Hillyard IW, Doczi J, Kiernan PB. Antacid and antiulcer properties of the polysaccharide chitosan in the rat. Proc Soc Exp Biol Med. 1964; 115:1108-1112.

108. Sugano M, Watanabe S, Kishi A, Izume M, Ohtakara A. Hypocholesterolemic action of chitosans with different viscosity in rats. Lipids. 1988;23(3):187-191

109. Kozen BG, Kircher SJ, Henao J, Godinez FS, Johnson AS. An alternative hemostatic dressing: comparison of CELOX, HemCon, and QuikClot. Acad Emerg Med. 2008;15(1):74-81.

110. Ueno H, Mori T, Fujinaga T. Topical formulations and wound healing applications of chitosan. Advanced Drug Delivery Reviews. 2001; 52(2):105-115.

111. Sashiwa H, Shigemasa Y. Chemical modification of chitin and chitosan 2: preparation and water soluble property of $\mathrm{N}$-acylated or $\mathrm{N}$-alkylated partially deacetylated chitins. Carbohydrate Polymers. 1999;39(2):127-138

112. Kaur L, Singh J, Liu Q. Starch - a potential biomaterial for biomedical applications. In: Mozafari MR, editor. Nanomaterials and Nanosystems for Biomedical Applications. Dordrecht: Springer; 2007: 83-98.

113. Calvert P. The structure of starch. Nature. 1997;389:338.

114. Betancur AD, Chel GL, Cañizares HE. Acetylation and Characterization of Canavalia ensiformis Starch. J Agri Food Chem. 1997; 45(2):378-382.

115. Salgado António J, Coutinho Olga P, Reis Rui L. Bone Tissue Engineering: State of the Art and Future Trends. Macromol Biosci. 2004;4(8): 743-765.

116. Mahdieh Z, Bagheri R, Eslami M, Amiri M, Shokrgozar MA, Mehrjoo M. Thermoplastic starch/ethylene vinyl alcohol/forsterite nanocomposite as a candidate material for bone tissue engineering. Mater Sci Eng C. 2016;69:301-310.

117. Ali Akbari Ghavimi S, Ebrahimzadeh Mohammad H, Solati-hashjin M, Abu Osman Noor A. Polycaprolactone/starch composite: Fabrication, structure, properties, and applications. J Biomed Mater Res A. 2014;103(7):2482-2498.

118. Averous L, Moro L, Dole P, Fringant C. Properties of thermoplastic blends: starch-polycaprolactone. Polymer. 2000;41(11):4157-4167.

119. Labet M, Thielemans W, Dufresne A. Polymer Grafting onto Starch Nanocrystals. Biomacromolecules. 2007;8(9):2916-2927. 
120. Wu KJ, Wu CS, Chang JS. Biodegradability and mechanical properties of polycaprolactone composites encapsulating phosphatesolubilizing bacterium Bacillus sp. PG01. Process Biochemistry. 2007;42(4):669-675.

121. Carvalho Pedro P, Leonor Isabel B, Smith Brenda J, et al. Undifferentiated human adipose-derived stromal/stem cells loaded onto wet-spun starch-polycaprolactone scaffolds enhance bone regeneration: Nude mice calvarial defect in vivo study. J Biomed Mater Res A. 2013;102(9):3102-3111.

122. Link Dennis P, Gardel Leandro S, Correlo Vitor M, Gomes Manuela E, Reis Rui L. Osteogenic properties of starch poly( $\varepsilon$-caprolactone) (SPCL) fiber meshes loaded with osteoblast-like cells in a rat critical-sized cranial defect. J Biomed Mater Res A. 2013;101(11):3059-3065.

123. Requicha Joao F, Moura T, Leonor Isabel B, et al. Evaluation of a starch-based double layer scaffold for bone regeneration in a rat model. J Orthop Res. 2014;32(7):904-909.

124. Vmd OC, Stringhetti Ferreira Cury B, Evangelista RC, Daflon Gremião MP. Development and characterization of cross-linked gellan gum and retrograded starch blend hydrogels for drug delivery applications. J Mech Behav Biomed Mater. 2017;65:317-333.

125. Dragan ES. Design and applications of interpenetrating polymer network hydrogels. A review. Chem Eng J. 2014;243:572-590.

126. Saboktakin MR, Tabatabaie RM, Maharramov A, Ramazanov MA. Synthesis and in vitro evaluation of carboxymethyl starch-chitosan nanoparticles as drug delivery system to the colon. Int J Biol Macromolecules. 2011;48(3):381-385.

127. Olivato JB, Müller CMO, Carvalho GM, Yamashita F, Grossmann MVE. Physical and structural characterisation of starch/polyester blends with tartaric acid. Mater Sci Eng C. 2014;39:35-39.

128. Pawar SN, Edgar KJ. Alginate derivatization: A review of chemistry, properties and applications. Biomaterials. 2012;33(11):3279-3305.

129. Lee KY, Mooney DJ. Alginate: Properties and biomedical applications. Progress Polymer Science. 2012;37(1):106-126.

130. Chandika P, Ko SC, Oh GW, et al. Fish collagen/alginate/chitooligosaccharides integrated scaffold for skin tissue regeneration application. Int J Biol Macromol. 2015;81:504-513.

131. Hajiali H, Heredia-Guerrero JA, Liakos I, Athanassiou A, Mele E. Alginate Nanofibrous Mats with Adjustable Degradation Rate for Regenerative Medicine. Biomacromolecules. 2015;16(3):936-943.

132. Ruvinov E, Cohen S. Alginate biomaterial for the treatment of myocardial infarction: Progress, translational strategies, and clinical outlook: From ocean algae to patient bedside. Adv Drug Deliv Rev. 2016;96: 54-76.

133. Kim HL, Jung GY, Yoon JH, et al. Preparation and characterization of nano-sized hydroxyapatite/alginate/chitosan composite scaffolds for bone tissue engineering. Mater Sci Eng C. 2015;54:20-25.

134. Sharma C, Dinda AK, Potdar PD, Chou C-F, Mishra NC. Fabrication and characterization of novel nano-biocomposite scaffold of chitosangelatin-alginate-hydroxyapatite for bone tissue engineering. Mater Sci Eng C. 2016;64:416-427.

135. Deng B, Shen L, Wu Y, et al. Delivery of alginate-chitosan hydrogel promotes endogenous repair and preserves cardiac function in rats with myocardial infarction. J Biomed Mater Res A. 2014;103(3): 907-918.

136. Boateng J, Catanzano O. Advanced Therapeutic Dressings for Effective Wound Healing\&\#x2014; A Review. J Pharm Sci. 2015;104(11): 3653-3680.

137. Boateng J, Burgos-Amador R, Okeke O, Pawar H. Composite alginate and gelatin based bio-polymeric wafers containing silver sulfadiazine for wound healing. Inte J Biol Macromolecules. 2015;79:63-71.

138. Tellechea A, Silva EA, Min J, et al. Alginate and DNA Gels Are Suitable Delivery Systems for Diabetic Wound Healing. Int $J$ Low Extrem Wounds. 2015;14(2):146-153.

139. Catanzano O, D’Esposito V, Acierno S, et al. Alginate-hyaluronan composite hydrogels accelerate wound healing process. Carbohydrate Polymers. 2015;131:407-414.
140. Contreras A, Fay D, Hanrahan K, Trevisone F. Review of article: Ozaki C, Hamdan A, Barshes N, Wyers M, Hevelone N, Belkin M, Nguyen L. Prospective, randomized, multi-institutional clinical trial of a silver alginate dressing to reduce lower-extremity vascular surgery wound complications. Society of vascular surgery 2015;61:419-427. J Vasc Nurs. 2016;34(2):59-60.

141. Venkatesan J, Bhatnagar I, Manivasagan P, Kang K-H, Kim S-K. Alginate composites for bone tissue engineering: A review. Int $\mathrm{J}$ Biol Macromolecules. 2015;72:269-281.

142. Ýa M, Donati I, Strand BL, Skjåk-Bræk G. Molecular Engineering as an Approach to Design New Functional Properties of Alginate. Biomacromolecules. 2007;8(9):2809-2814.

143. Altman GH, Diaz F, Jakuba C, et al. Silk-based biomaterials. Biomaterials. 2003;24(3):401-416.

144. Cao T-T, Zhang Y-Q. Processing and characterization of silk sericin from Bombyx mori and its application in biomaterials and biomedicines. Mater Sci Eng C. 2016;61:940-952.

145. Jao D, Mou X, Hu X. Tissue Regeneration: A Silk Road. J Funct Biomaterials. 2016;7(3):22.

146. Giesa T, Arslan M, Pugno NM, Buehler MJ. Nanoconfinement of Spider Silk Fibrils Begets Superior Strength, Extensibility, and Toughness. Nano Lett. 2011;11(11):5038-5046.

147. Chawla S, Midha S, Sharma A, Ghosh S. Silk-Based Bioinks for 3D Bioprinting. Adv Healthcare Mater. 2018;7(8):1701204.

148. Kundu B, Rajkhowa R, Kundu SC, Wang X. Silk fibroin biomaterials for tissue regenerations. Adv Drug Deliv Rev. 2013;65(4):457-470.

149. Shao W, He J, Sang F, et al. Coaxial electrospun aligned tussah silk fibroin nanostructured fiber scaffolds embedded with hydroxyapatitetussah silk fibroin nanoparticles for bone tissue engineering. Mater Sci Eng C Mater Biol Appl. 2016;58:342-351.

150. Tian L, Prabhakaran MP, Hu J, Chen M, Besenbacher F, Ramakrishna S. Coaxial electrospun poly(lactic acid)/silk fibroin nanofibers incorporated with nerve growth factor support the differentiation of neuronal stem cells. RSC Advances. 2015;5(62):49838-49848.

151. Bhardwaj N, Singh YP, Devi D, Kandimalla R, Kotoky J, Mandal BB. Potential of silk fibroin/chondrocyte constructs of muga silkworm Antheraea assamensis for cartilage tissue engineering. $J$ Mater Chem B. 2016;4(21):3670-3684.

152. Bhardwaj N, Sow WT, Devi D, Kw N, Mandal BB, Cho N-J. Silk fibroin-keratin based 3D scaffolds as a dermal substitute for skin tissue engineering. Integrative Biology. 2015;7(1):53-63.

153. Font Tellado S, Chiera S, Bonani W, et al. Heparin functionalization increases retention of TGF- $\beta 2$ and GDF5 on biphasic silk fibroin scaffolds for tendon/ligament-to-bone tissue engineering. Acta Biomaterialia. 2018;72:150-166.

154. Gil Eun S, Panilaitis B, Bellas E, Kaplan David L. Functionalized Silk Biomaterials for Wound Healing. Adv Healthcare Mater. 2012;2(1): 206-217.

155. Min S, Gao X, Han C, et al. Preparation of a silk fibroin spongy wound dressing and its therapeutic efficiency in skin defects. J Biomater Sci Polym Ed. 2012;23(1-4):97-110.

156. Zhang W, Chen L, Chen J, et al. Silk Fibroin Biomaterial Shows Safe and Effective Wound Healing in Animal Models and a Randomized Controlled Clinical Trial. Adv Healthcare Mater. 2017;6(10): 1700121.

157. Zhou Y, Yang H, Liu X, Mao J, Gu S, Xu W. Electrospinning of carboxyethyl chitosan/poly(vinyl alcohol)/silk fibroin nanoparticles for wound dressings. Int J Biol Macromol. 2013;53:88-92.

158. Chutipakdeevong J, Ruktanonchai U, Supaphol P. Hybrid biomimetic electrospun fibrous mats derived from poly( $\varepsilon$-caprolactone) and silk fibroin protein for wound dressing application. J Appl Polymer Sci. 2014;132(11).

159. Uttayarat $P$, Jetawattana $S$, Suwanmala $P$, Eamsiri J, Tangthong T, Pongpat S. Antimicrobial electrospun silk fibroin mats with silver nanoparticles for wound dressing application. Fibers Polymers. 2012; 13(8):999-1006 
160. Gu Z, Xie H, Huang C, Li L, Yu X. Preparation of chitosan/silk fibroin blending membrane fixed with alginate dialdehyde for wound dressing. Int J Biol Macromol. 2013;58:121-126.

161. Guang S, An Y, Ke F, Zhao D, Shen Y, Xu H. Chitosan/silk fibroin composite scaffolds for wound dressing. J Appl Polymer Sci. 2015; 132(35).

162. Wenk E, Merkle HP, Meinel L. Silk fibroin as a vehicle for drug delivery applications. J Control Release. 2011;150(2):128-141.

163. Mottaghitalab F, Farokhi M, Shokrgozar MA, Atyabi F, Hosseinkhani H. Silk fibroin nanoparticle as a novel drug delivery system. J Control Release. 2015;206:161-176.

164. Hofer M, Winter G, Myschik J. Recombinant spider silk particles for controlled delivery of protein drugs. Biomaterials. 2012;33(5): 1554-1562.

165. Pritchard EM, Valentin T, Panilaitis B, Omenetto F, Kaplan DL, Pritchard Eleanor M, Kaplan David L. Antibiotic-Releasing Silk Biomaterials for Infection Prevention and Treatment. Adv Funct Mater. 2013;23(7):854-861.

166. Seib FP, Jones GT, Rnjak-Kovacina J, Lin Y, Kaplan DL, Jones Gregory T, Kaplan David L. pH-dependent anticancer drug release from silk nanoparticles. Adv Healthc Mater. 2013;2(12):1606-1611.

167. Ozbolat IT. Bioprinting scale-up tissue and organ constructs for transplantation. Trends Biotechnol. 2015;33(7):395-400.

168. Holzl K, Lin S, Tytgat L, van Vlierberghe S, Gu L, Ovsianikov A. Bioink properties before, during and after 3D bioprinting. Biofabrication. 2016;8(3):032002

169. Schacht K, Jungst T, Schweinlin M, Ewald A, Groll J, Scheibel T. Biofabrication of cell-loaded 3D spider silk constructs. Angewandte Chemie (International ed. in English). 2015;54(9):2816-2820.

170. Billiet T, Gevaert E, de Schryver T, Cornelissen M, Dubruel P. The $3 \mathrm{D}$ printing of gelatin methacrylamide cell-laden tissue-engineered constructs with high cell viability. Biomaterials. 2014;35(1):49-62.

171. Hood EE, Jilka JM. Plant-based production of xenogenic proteins. Curr Opin Biotechnol. 1999;10(4):382-386.

172. Lazaris A, Arcidiacono S, Huang Y. Spider silk fibers spun from soluble recombinant silk produced in mammalian cells. Science (New York, N. Y.). 2002;295(5554):472-476.

173. Lamboni L, Gauthier M, Yang G, Wang Q. Silk sericin: A versatile material for tissue engineering and drug delivery. Biotechnology Advances. 2015;33(8):1855-1867.

174. Aramwit P, Siritientong T, Kanokpanont S, Srichana T. Formulation and characterization of silk sericin-PVA scaffold crosslinked with genipin. Int J Biol Macromol. 2010;47(5):668-675.

175. Chen GQ. A microbial polyhydroxyalkanoates (PHA) based bio- and materials industry. Chem Soc Rev. 2009;38(8):2434-2446.

176. Li Z, Loh XJ. Recent advances of using polyhydroxyalkanoate-based nanovehicles as therapeutic delivery carriers. Wiley Interdiscip Rev Nanomed Nanobiotechnol. 2017;9(3):e1429-n/a:e1429.

177. Chen GQ, Wu Q. The application of polyhydroxyalkanoates as tissue engineering materials. Biomaterials. 2005;26(33):6565-6578.

178. Andrade AP, Witholt B, Chang D, Li Z. Synthesis and Characterization of Novel Thermoplastic Polyester Containing Blocks of Poly[(R)-3hydroxyoctanoate] and Poly[(R)-3-hydroxybutyrate]. Macromolecules. 2003;36(26):9830-9835.

179. Li Z, Yang J, Loh XJ. Polyhydroxyalkanoates: opening doors for a sustainable future. NPG Asia Mater. 2016;8:e265.

180. Hazer B, Steinbuchel A. Increased diversification of polyhydroxyalkanoates by modification reactions for industrial and medical applications. Appl Microbiol Biotechnol. 2007;74(1):1-12.

181. Saito Y, Doi Y. Microbial synthesis and properties of poly(3-hydroxybutyrate-co-4-hydroxybutyrate) in Comamonas acidovorans. Int $J$ Biol Macromol. 1994;16(2):99-104.

182. Park SJ, Ahn WS, Green PR, Lee SY. Biosynthesis of poly(3hydroxybutyrate-co-3-hydroxyvalerate-co-3-hydroxyhexanoate) by metabolically engineered Escherichia coli strains. Biotechnol Bioeng. 2001;74(1):82-87.
183. Kai D, Liow SS, Loh XJ. Biodegradable polymers for electrospinning: towards biomedical applications. Mater Sci Eng C Mater Biol Appl. 2014;45(Supplement C):659-670.

184. Lim J, You M, Li J, Li Z. Emerging bone tissue engineering via Polyhydroxyalkanoate (PHA)-based scaffolds. Mater Sci Eng C Mater Biol Appl. 2017;79:917-929.

185. Bagdadi AV, Safari M, Dubey P, et al. Poly(3-hydroxyoctanoate), a promising new material for cardiac tissue engineering. J Tissue Eng Regen Med. 2018 Jan;12(1):e495-e512.

186. Shishatskaya EI, Nikolaeva ED, Vinogradova ON, Volova TG. Experimental wound dressings of degradable PHA for skin defect repair. J Mater Sci Mater Med. 2016;27(11):165.

187. Goonoo N, Bhaw-Luximon A, Passanha P, Esteves SR, Jhurry D. Third generation poly(hydroxyacid) composite scaffolds for tissue engineering. J Biomed Mater Res B Appl Biomater. 2017;105(6):1667-1684.

188. Loh XJ, Wang X, Li H, Li X, Li J. Compositional study and cytotoxicity of biodegradable poly(ester urethane)s consisting of poly[(R)-3hydroxybutyrate] and poly(ethylene glycol). Mater Science Eng C. 2007;27(2):267-273

189. Loh XJ, Goh SH, Li J. Hydrolytic degradation and protein release studies of thermogelling polyurethane copolymers consisting of poly[(R)-3-hydroxybutyrate], poly(ethylene glycol), and poly(propylene glycol). Biomaterials. 2007;28(28):4113-4123.

190. Y-L W, Wang H, Qiu Y-K, Loh XJ. PLA-based thermogel for the sustained delivery of chemotherapeutics in a mouse model of hepatocellular carcinoma. RSC Advances. 2016;6(50):44506-44513.

191. Yum MS, Ts K, Kim DW. Beta-Hydroxybutyrate increases the pilocarpine-induced seizure threshold in young mice. Brain Dev. 2012;34(3):181-184.

192. Cheng S, Wu Q, Yang F, Xu M, Leski M, Chen GQ. Influence of DL-beta-hydroxybutyric acid on cell proliferation and calcium influx. Biomacromolecules. 2005;6(2):593-597.

193. Maalouf M, Sullivan PG, Davis L, Kim DY, Rho JM. Ketones inhibit mitochondrial production of reactive oxygen species production following glutamate excitotoxicity by increasing NADH oxidation. Neuroscience. 2007;145(1):256-264.

194. Wu Q, Wang Y, Chen G-Q. Medical Application of Microbial Biopolyesters Polyhydroxyalkanoates. Artif Cells Blood Substit Immobil Biotechnol. 2009;37(1):1-12.

195. Strong PJ, Laycock B, Mahamud SN, et al. The Opportunity for High-Performance Biomaterials from Methane. Microorganisms. 2016;4(1).

196. Koller M, Maršálek L, de Sousa Dias MM, Braunegg G. Producing microbial polyhydroxyalkanoate (PHA) biopolyesters in a sustainable manner. New Biotechnology. 2017;37(Part A):24-38.

197. Huang Y, Wang Y, Sun L, Agrawal R, Zhang M. Sundew adhesive: a naturally occurring hydrogel. J R Soc Interface. 2015;12(107): 20150226.

198. Rost K, Schauer R. Physical and chemical properties of the mucin secreted by Drosera capensis. Phytochemistry. 1977;16(9):1365-1368.

199. Zhang M, Lenaghan SC, Xia L, et al. Nanofibers and nanoparticles from the insect-capturing adhesive of the Sundew (Drosera) for cell attachment. J Nanobiotechnology. 2010;8:20.

200. Sun L, Huang Y, Bian Z, et al. Sundew-Inspired Adhesive Hydrogels Combined with Adipose-Derived Stem Cells for Wound Healing. ACS Appl Mater Interfaces. 2016;8(3):2423-2434.

201. Zhang M, Liu M, Prest H, Fischer S. Nanoparticles Secreted from Ivy Rootlets for Surface Climbing. Nano Lett. 2008;8(5):1277-1280.

202. Lenaghan SC, Burris JN, Chourey K, et al. Isolation and chemical analysis of nanoparticles from English ivy (Hedera helix L). J R Soc Interface. 2013;10(87):20130392.

203. Wu Y, Zhao X, Zhang M. Adhesion mechanics of ivy nanoparticles. $J$ Colloid Interface Sci. 2010;344(2):533-540.

204. Li Q, Xia L, Zhang Z, Zhang M. Ultraviolet Extinction and Visible Transparency by Ivy Nanoparticles. Nanoscale Res Lett. 2010;5(9): 1487-1491. 
205. Xia L, Lenaghan SC, Zhang M, Zhang Z, Li Q. Naturally occurring nanoparticles from English ivy: an alternative to metal-based nanoparticles for UV protection. J Nanobiotechnology. 2010;8(1):12.

206. Schmidt CE, Baier JM. Acellular vascular tissues: natural biomaterials for tissue repair and tissue engineering. Biomaterials. 2000; 21(22):2215-2231

207. Seal B, Otero TC, Panitch A. Polymeric biomaterials for tissue and organ regeneration. Mater Sci Eng Rep. 2001;34(4-5):147-230.

208. Fournier E, Passirani C, Montero-Menei CN, Benoit JP. Biocompatibility of implantable synthetic polymeric drug carriers: focus on brain biocompatibility. Biomaterials. 2003;24(19):3311-3331.

209. Zheng Z, Yin W, Zara JN, et al. The use of BMP-2 coupled-NanosilverPLGA composite grafts to induce bone repair in grossly infected segmental defects. Biomaterials. 2010;31(35):9293-9300.

210. Liu Y, Zheng Z, Zara JN, et al. The antimicrobial and osteoinductive properties of silver nanoparticle/poly (DL-lactic-co-glycolic acid)coated stainless steel. Biomaterials. 2012;33(34):8745-8756.

211. Bernardini G, Chellini F, Frediani B, Spreafico A, Santucci A. Human platelet releasates combined with polyglycolic acid scaffold promote chondrocyte differentiation and phenotypic maintenance. J Biosci. 2015;40(1):61-69.

212. Cameron DJ, Shaver MP. Aliphatic polyester polymer stars: synthesis, properties and applications in biomedicine and nanotechnology. Chem Soc Rev. 2011;40(3):1761-1776.

213. Mano JF, Sousa RA, Boesel LF, Neves NM, Bioinert RRL. Biodegradable and injectable polymeric matrix composites for hard tissue replacement: state of the art and recent developments. Composit Sci Technol. 2004;64(6):789-817.

214. Seyednejad H, Ghassemi AH, van Nostrum CF, Vermonden T, Hennink WE. Functional aliphatic polyesters for biomedical and pharmaceutical applications. J Control Release. 2011;152(1):168-176.

215. Buescher JM, Margaritis A. Microbial biosynthesis of polyglutamic acid biopolymer and applications in the biopharmaceutical, biomedical and food industries. Crit Rev Biotechnol. 2007;27(1):1-19.

216. Multani AS, Li C, Ozen M, et al. Paclitaxel and water-soluble poly (L-glutamic acid)-paclitaxel, induce direct chromosomal abnormalities and cell death in a murine metastatic melanoma cell line. Anticancer Res. 1997;17(6D):4269-4274.

217. Li C, Ke S, Wu QP, Qp W, et al. Tumor irradiation enhances the tumor-specific distribution of poly(L-glutamic acid)-conjugated paclitaxel and its antitumor efficacy. Clin Cancer Res. 2000;6(7): 2829-2834.

218. Iwata H, Matsuda S, Mitsuhashi K, Itoh E, Ikada Y. A novel surgical glue composed of gelatin and N-hydroxysuccinimide activated poly(L-glutamic acid): Part 1. Synthesis of activated poly(L-glutamic acid) and its gelation with gelatin. Biomaterials. 1998;19(20): 1869-1876.

219. Otani Y, Tabata Y, Ikada Y. Rapidly curable biological glue composed of gelatin and poly(L-glutamic acid). Biomaterials. 1996;17(14): $1387-1391$.

220. Takeuchi J, Suzuki H, Murata M, et al. Clinical evaluation of application of polyglycolic acid sheet and fibrin glue spray for partial glossectomy. J Oral Maxillofac Surg. 2013;71(2):e126-e131.

221. Shinozaki T, Hayashi R, Ebihara M, Miyazaki M, Tomioka T. Mucosal defect repair with a polyglycolic acid sheet. Jpn J Clin Oncol. 2013;43(1):33-36.

222. Rokutanda S, Yanamoto S, Yamada S, Naruse T, Inokuchi S, Umeda M. Application of polyglycolic acid sheets and fibrin glue spray to bone surfaces during oral surgery: a case series. J Oral Maxillofac Surg. 2015;73(5):1017.e1-101017.e1.

223. Maurus PB, Kaeding CC. Bioabsorbable implant material review. Oper Tech Sports Med. 2004;12(3):158-160.

224. Llorens E, Calderón S, del Valle LJ, Puiggalí J, Polybiguanide PJ. Polybiguanide (PHMB) loaded in PLA scaffolds displaying high hydrophobic, biocompatibility and antibacterial properties. Mater Sci Eng C Mater Biol Appl. 2015;50:74-84.
225. Dong H, Dai W, Ju H, et al. Multifunctional Poly(L-lactide)-Polyethylene Glycol-Grafted Graphene Quantum Dots for Intracellular MicroRNA Imaging and Combined Specific-Gene-Targeting Agents Delivery for Improved Therapeutics. ACS Appl Mater Interfaces. 2015;7(20):11015-11023.

226. Gollwitzer H, Thomas P, Diehl P, et al. Biomechanical and allergological characteristics of a biodegradable poly(D,L-lactic acid) coating for orthopaedic implants. J Orthop Res. 2005;23(4):802-809.

227. Schmidmaier G, Wildemann B, Stemberger A, Haas NP, Raschke M. Biodegradable poly(D,L-lactide) coating of implants for continuous release of growth factors. J Biomed Mater Res. 2001;58(4): 449-455.

228. Félix Lanao RP, Jonker AM, Wolke JG, Jansen JA, van Hest JC, Leeuwenburgh SC. Physicochemical properties and applications of poly(lactic-co-glycolic acid) for use in bone regeneration. Tissue Eng Part B Rev. 2013;19(4):380-390.

229. Pan Z, Ding J, Poly DJ. Poly(lactide-co-glycolide) porous scaffolds for tissue engineering and regenerative medicine. Interface Focus. 2012;2(3):366-377.

230. Shi X, Wang Y, Ren L, Gong Y, Wang DA. Enhancing alendronate release from a novel PLGA/hydroxyapatite microspheric system for bone repairing applications. Pharm Res. 2009;26(2):422-430.

231. Labet M, Thielemans W. Synthesis of polycaprolactone: a review. Chem Soc Rev. 2009;38(12):3484-3504.

232. Baradaran-Rafii A, Biazar E, Heidari-Keshel S. Cellular Response of Limbal Stem Cells on Polycaprolactone Nanofibrous Scaffolds for Ocular Epithelial Regeneration. Curr Eye Res. 2016;41(3):1-8.

233. Konopnicki S, Sharaf B, Resnick C, et al. Tissue-Engineered Bone With 3-Dimensionally Printed $\beta$-Tricalcium Phosphate and Polycaprolactone Scaffolds and Early Implantation: An In Vivo Pilot Study in a Porcine Mandible Model. J Oral Maxillofac Surg. 2015; 73(5):1016.e1-101016.e1.

234. Mcneil SE, Griffiths HR, Perrie Y. Polycaprolactone fibres as a potential delivery system for collagen to support bone regeneration. Curr Drug Deliv. 2011;8(4):448-455.

235. Yildirim ED, Ayan H, Vasilets VN, Fridman A, Guceri S, Sun W. Effect of Dielectric Barrier Discharge Plasma on the Attachment and Proliferation of Osteoblasts Cultured over Poly( $\varepsilon$-caprolactone) Scaffolds. Plasma Process Polym. 2008;5(1):58-66.

236. Singh RK, Jin GZ, Mahapatra C, Patel KD, Chrzanowski W, Kim HW. Mesoporous silica-layered biopolymer hybrid nanofibrous scaffold: a novel nanobiomatrix platform for therapeutics delivery and bone regeneration. ACS Appl Mater Interfaces. 2015;7(15): 8088-8098.

237. Sinha VR, Bansal K, Kaushik R, Kumria R, Trehan A. Poly-epsiloncaprolactone microspheres and nanospheres: an overview. Int J Pharm. 2004;278(1):1-23.

238. Mondrinos MJ, Dembzynski R, Lu L, et al. Porogen-based solid freeform fabrication of polycaprolactone-calcium phosphate scaffolds for tissue engineering. Biomaterials. 2006;27(25):4399-4408.

239. Chiari C, Koller U, Dorotka R, et al. A tissue engineering approach to meniscus regeneration in a sheep model. Osteoarthritis Cartilage. 2006;14(10):1056-1065.

240. Jain JP, Chitkara D, Kumar N. Polyanhydrides as localized drug delivery carrier: an update. Expert Opin Drug Deliv. 2008;5(8):889-907.

241. Jain JP, Modi S, Domb AJ, Kumar N. Role of polyanhydrides as localized drug carriers. J Control Release. 2005;103(3):541-563.

242. Katti DS, Lakshmi S, Langer R, Laurencin CT, Toxicity LCT. Toxicity, biodegradation and elimination of polyanhydrides. Adv Drug Deliv Rev. 2002;54(7):933-961.

243. Torres MP, Vogel BM, Narasimhan B, Mallapragada SK. Synthesis and characterization of novel polyanhydrides with tailored erosion mechanisms. J Biomed Mater Res A. 2006;76(1):102-110.

244. Li LC, Deng J, Stephens D. Polyanhydride implant for antibiotic delivery - from the bench to the clinic. Adv Drug Deliv Rev. 2002; 54(7):963-986. 
245. Santerre JP, Woodhouse K, Laroche G, Labow RS. Understanding the biodegradation of polyurethanes: from classical implants to tissue engineering materials. Biomaterials. 2005;26(35):7457-7470.

246. Guelcher SA. Biodegradable polyurethanes: synthesis and applications in regenerative medicine. Tissue Eng Part B Rev. 2008;14(1):3-17.

247. Zhang JY, Doll BA, Beckman EJ, Hollinger JO. Three-dimensional biocompatible ascorbic acid-containing scaffold for bone tissue engineering. Tissue Eng. 2003;9(6):1143-1157.

248. Saad B, Hirt TD, Welti M, Uhlschmid GK, Neuenschwander P, Suter UW. Development of degradable polyesterurethanes for medical applications: in vitro and in vivo evaluations. J Biomed Mater Res. 1997;36(1):65-74.

249. Bonzani IC, Adhikari R, Houshyar S, Mayadunne R, Gunatillake P, Stevens MM. Synthesis of two-component injectable polyurethanes for bone tissue engineering. Biomaterials. 2007;28(3):423-433.

250. Solanki A, Thakore S. Cellulose crosslinked $\mathrm{pH}$-responsive polyurethanes for drug delivery: $\alpha$-hydroxy acids as drug release modifiers. Int J Biol Macromol. 2015;80:683-691.

251. Nair LS, Laurencin CT. Polymers as biomaterials for tissue engineering and controlled drug delivery. Adv Biochem Eng Biotechnol. 2006;102:47-90.

252. Laurencin CT, Norman ME, Elgendy HM, et al. Use of polyphosphazenes for skeletal tissue regeneration. J Biomed Mater Res. 1993;27(7): 963-973.

253. Ambrosio AM, Allcock HR, Katti DS, Laurencin CT. Degradable polyphosphazene/poly(alpha-hydroxyester) blends: degradation studies. Biomaterials. 2002;23(7):1667-1672.
254. Lakshmi S, Katti DS, Laurencin CT. Biodegradable polyphosphazenes for drug delivery applications. Adv Drug Deliv Rev. 2003;55(4): 467-482.

255. Nair LS, Lee DA, Bender JD, et al. Synthesis, characterization, and osteocompatibility evaluation of novel alanine-based polyphosphazenes. J Biomed Mater Res A. 2006;76(1):206-213.

256. Kumbar SG, Bhattacharyya S, Nukavarapu SP, Khan YM, Nair LS, Laurencin CT. In Vitro and In Vivo Characterization of Biodegradable Poly(organophosphazenes) for Biomedical Applications. J Inorg Organomet Polym Mater. 2007;16(4):365-385.

257. Sethuraman S, Nair LS, El-Amin S, et al. In vivo biodegradability and biocompatibility evaluation of novel alanine ester based polyphosphazenes in a rat model. J Biomed Mater Res A. 2006;77(4):679-687.

258. Greish YE, Bender JD, Lakshmi S, Brown PW, Allcock HR, Laurencin CT. Low temperature formation of hydroxyapatitepoly(alkyl oxybenzoate)phosphazene composites for biomedical applications. Biomaterials. 2005;26(1):1-9.

259. Rothemund S, Aigner TB, Iturmendi A, et al. Degradable glycinebased photo-polymerizable polyphosphazenes for use as scaffolds for tissue regeneration. Macromol Biosci. 2015;15(3):351-363.

260. Krogman NR, Weikel AL, Kristhart KA, et al. The influence of side group modification in polyphosphazenes on hydrolysis and cell adhesion of blends with PLGA. Biomaterials. 2009;30(17):3035-3041.
Drug Design, Development and Therapy

\section{Publish your work in this journal}

Drug Design, Development and Therapy is an international, peerreviewed open-access journal that spans the spectrum of drug design and development through to clinical applications. Clinical outcomes, patient safety, and programs for the development and effective, safe, and sustained use of medicines are the features of the journal, which

\section{Dovepress}

has also been accepted for indexing on PubMed Central. The manuscript management system is completely online and includes a very quick and fair peer-review system, which is all easy to use. Visit http://www.dovepress.com/testimonials.php to read real quotes from published authors.

Submit your manuscript here: http://www.dovepress.com/drug-design-development-and-therapy-journal 